\title{
Factors associated with self-reported anxiety, depression, and general health during the UK lockdown; a cross- sectional survey
}

Louise E. Smith ${ }^{1,2}$, PhD, Richard Amlot ${ }^{2,3,4}, \mathrm{PhD}$, Helen Lambert ${ }^{4,5}$, PhD, Isabel Oliver ${ }^{4,5,6}$, PhD, Charlotte Robin ${ }^{4,6,7,8}$, PhD, Lucy Yardley ${ }^{4,9,10}$, PhD, G James Rubin ${ }^{1,2}$, PhD

1 King's College London, Institute of Psychiatry, Psychology and Neuroscience

2 NIHR Health Protection Research Unit in Emergency Preparedness and Response

3 Public Health England, Behavioural Science Team, Emergency Response Department

Science and Technology

4 NIHR Health Protection Research Unit in Behavioural Science and Evaluation, University of Bristol

5 University of Bristol, Department of Population Health Sciences, Bristol Medical School

6 Public Health England, Field Epidemiology, Field Service, National Infection Service

7 NIHR Health Protection Research Unit in Emerging and Zoonotic Infections

8 NIHR Health Protection Research Unit in Gastrointestinal Infections

9 University of Bristol, School of Psychological Science

10 University of Southampton, Department of Psychology

Corresponding author: Louise E. Smith, Post-doctoral Researcher. Department of

Psychological Medicine, King's College London, Weston Education Centre, Cutcombe Road, London, SE5 9RJ. Email: louise.e.smith@kcl.ac.uk

Richard Aml $\square \mathrm{t}$, Head of Behavioural Science in the Emergency Response Department at Public Health England. 
medRxiv preprint doi: https://doi.org/10.1101/2020.06.23.20137901; this version posted June 23, 2020. The copyright holder for this preprint (which was not certified by peer review) is the author/funder, who has granted medRxiv a license to display the preprint in perpetuity.

It is made available under a CC-BY 4.0 International license .

Helen Lambert, Professor of Medical Anthropology. Department of Population Health

Sciences, Canynge Hall, 39 Whatley Road, Bristol BS8 2PS.

Isabel Oliver, Director of the Field Epidemiology Service of Public Health England. Field

Epidemiology, Field Service, National Infection Service, Public Health England, Bristol, BS1 $6 \mathrm{EH}$.

Charlotte Robin, Field Epidemiology, Field Service, National Infection Service, Public

Health England, Bristol, BS1 6EH.

Lucy Yardley, Professor of Health Psychology. School of Psychological Science, 12A Priory

Road, Clifton, Bristol, BS8 1TU.

G James Rubin, Reader in the Psychology of Emerging Health Risks. Department of

Psychological Medicine, King's College London, Weston Education Centre, Cutcombe Road, London, SE5 9RJ.

\section{AUTHOR CONTRIBUTION STATEMENT}

The study was conceptualised by RA, HL, IO, CR, LY and GJR. LS completed all analyses, using data from YouGov Plc. All authors contributed to, and approved, the final manuscript. For any enquiries about the data in this report please contact King's College London. 


\section{RESEARCH IN CONTEXT}

\section{Evidence before this study}

- Quarantine is associated with adverse psychological outcomes.

- Psychological distress during quarantine is associated with greater financial loss, greater perceived susceptibility to and severity of the illness, and greater frustration and boredom during quarantine.

- Measures put in place to prevent the spread of COVID-19 have highlighted existing inequalities in society, disproportionally affecting younger people, those in lowerincome households, and Black and minority ethnic groups.

- Research in the UK and other countries indicates that rates of anxiety and depression during restrictions of movement such as "lockdown" measures are higher than population norms.

\section{Added value of this study}

- In this study, $22 \%$ of the sample reported anxiety, while $24 \%$ reported depression. Normative data indicate that these rates are usually approximately $5 \%$ and $7 \%$ respectively.

- Factors associated with psychological distress included greater financial hardship, poorer social connectedness, greater conflict within the household and the wider neighbourhood, being female and of younger age.

- Self-reported general health in the sample was "good" on average. Factors associated with poorer self-reported general health included markers of inequality and greater worry about COVID-19.

\section{Implications of all the available evidence}

- Decreasing the financial impact of measures put in place to prevent the spread of COVID-19 may help improve mental health.

- Interventions promoting social connectedness in isolated young people and measures that increase household and neighbourhood solidarity may help improve mental health. 
medRxiv preprint doi: https://doi.org/10.1101/2020.06.23.20137901; this version posted June 23, 2020. The copyright holder for this preprint (which was not certified by peer review) is the author/funder, who has granted medRxiv a license to display the preprint in perpetuity.

It is made available under a CC-BY 4.0 International license .

\begin{abstract}
Background: To investigate factors associated with anxiety, depression, and self-reported general health during "lockdown" due to COVID-19 in the UK.

Methods: Online cross-sectional survey of a nationally-representative sample of 2240

participants living in the UK aged 18 years or over (data collected 6-7 May 2020).

Participants were recruited from YouGov's online research panel.

Outcomes: In this sample, $21.9 \%(\mathrm{n}=458,95 \%$ CI [20.1\% to $23.7 \%])$ reported probable

anxiety (scored three or over on the GAD-2); while $23 \cdot 5 \%(n=494,95 \%$ CI [21.7\% to 25.3]) reported probable depression (scored three or over on the PHQ-2). Poorer mental health was associated with greater financial hardship during the lockdown, thinking that you would lose contact with friends or family if you followed Government measures, more conflict with household members during the lockdown, less sense of community with people in your neighbourhood, and lower perceived effectiveness of Government measures. Females and those who were younger were likely to report higher levels of anxiety and depression. The majority of participants reported their general health as "good" (as measured by the first item of the SF-36). Poorer self-reported general health was associated with psychological distress, greater worry about COVID-19 and markers of inequality.

Interpretation: Rates of self-reported anxiety and depression in the UK during the lockdown were greater than population norms. Reducing financial hardship, promoting social connectedness, and increasing solidarity with neighbours and household members may help ease rifts within the community which are associated with distress, thereby improving mental health. Reducing inequality may also improve general health.
\end{abstract}

Key words: COVID-19; mental health; psychological distress; physical health 
medRxiv preprint doi: https://doi.org/10.1101/2020.06.23.20137901; this version posted June 23, 2020. The copyright holder for this preprint (which was not certified by peer review) is the author/funder, who has granted medRxiv a license to display the preprint in perpetuity.

It is made available under a CC-BY 4.0 International license.

\section{INTRODUCTION}

The COVID-19 pandemic has seen unprecedented levels of restrictions of movement worldwide. Quarantine has negative psychological consequences. ${ }^{1}$ Psychological distress from quarantine is associated with situational factors such as longer duration of quarantine, having inadequate supplies (such as food, clothes, or accommodation) and greater financial loss, and psychological factors, such as greater perceived susceptibility to and severity of the illness, frustration, boredom, inadequate information, and stigmatisation. ${ }^{1}$ The UK Government imposed "lockdown" measures restricting people's movement apart from for limited, specified reasons on $23^{\text {rd }}$ March $2020,{ }^{2}$ which were slightly eased on $11^{\text {th }}$ May $2020 .^{3}$ The pandemic has highlighted inequalities already present in society. For example, evidence shows that measures put in place to prevent the spread of COVID-19 have disproportionally affected females, younger people, those in low-income households and Black and minority ethnic groups. ${ }^{4}$

Research is beginning to emerge indicating that those under lockdown measures have higher levels of psychological distress. ${ }^{5-8}$ In the UK, preliminary research has shown that average levels of anxiety, depression and stress were higher during the lockdown than population norms. ${ }^{9}$ However, these results should be taken with caution as the sample was not demographically representative of the UK population (e.g. $85 \%$ female) and data were collected across almost four weeks. Results from a demographically-representative sample of the UK population show that anxiety was associated with loneliness, female sex, and one's work being affected by the COVID-19 pandemic. ${ }^{10}$ However, anxiety was not measured using a validated scale. While reported levels of mental distress should be taken with caution in rapidly constructed samples, ${ }^{11}$ where studies are rigorously conducted, associations within the data may still provide useful insights. ${ }^{12}$ Rapid research undertaken during public health crises often results in decreased methodological rigour. ${ }^{12,13}$ Online quota sampling is a standard approach used in opinion polling and can be a pragmatic approach when a large, demographically representative sample must be obtained in a very short time frame, particularly during a crisis. ${ }^{12}$

In this study, we investigated psychological, situational, personal and clinical factors associated with validated measures of anxiety and depression, and self-reported general health in a demographically representative sample of the UK adult population during lockdown. 


\section{METHOD}

\section{Design}

We commissioned YouGov Plc, a market research company, to carry out this cross-sectional survey, between $6^{\text {th }}$ and $7^{\text {th }}$ May 2020.

Results from analyses investigating adherence to self-isolation and lockdown measures using the same sample have been reported elsewhere. ${ }^{14,15}$

\section{Participants}

Participants ( $n=2240)$ were recruited from YouGov's online research panel ( $n=800,000+$ UK adults) and were eligible for the study if they were aged eighteen years or over and lived in the UK. To ensure that the sample was broadly representative of the UK general population, we used quota sampling. Quotas were set based on age, gender, social grade and highest level of education, and Government Office Region. Of 2,623 people who started the survey, 2,314 completed it. Seventy-four people were excluded from the sample as data were missing for demographic questions, or their response did not meet quality control standards (completed the survey suspiciously quickly or gave identical answers for multiple consecutive questions). Participants who completed the survey were reimbursed in points equivalent to approximately 50 pence.

\section{Study materials}

Full survey materials are available in the supplementary materials.

Outcome measures

We measured probable anxiety using the validated GAD-2. ${ }^{16}$ We measured probable depression using the validated PHQ-2. ${ }^{17}$ For these items, participants were asked to rate how much they had been bothered in the past two weeks on a four-point Likert-type scale from "not at all" to "nearly every day". Participants could also answer "don't know" and "prefer not to say" to these questions.

Participants rated their general health on a five-point Likert-type scale from "poor" to "excellent" using the first question from the SF-36. ${ }^{18}$

Psychological and situational factors

We asked participants whether they thought they "had, or currently have, coronavirus". Possible answers were "I have definitely had it or definitely have it now", "I have probably 
medRxiv preprint doi: https://doi.org/10.1101/2020.06.23.20137901; this version posted June 23, 2020. The copyright holder for this preprint

had it or probably have it now", "I have probably not had it and probably don't have it now", and "I have definitely not had it and definitely don't have it now".

Participants reported whether they had experienced symptoms "in the past seven days" from a list of thirteen symptoms including cough and high temperature / fever. Participants who lived with someone else also reported if "someone else in [their] household" had experienced symptoms "in the past fourteen days" from the same list of thirteen symptoms.

Participants were asked to indicate whether they were currently self-isolating, choosing between "not self-isolating", "self-isolating for seven days", "self-isolating for fourteen days", and "self-isolating for at least 12 weeks".

Participants reported "in the past seven days, how many times, if at all, [they had] left [their] home" to go to the shops for groceries, toiletries or medicine, to go to the shops for other items, for exercise, for a medical purpose, to go to work, and to help someone else.

We asked participants how worried they were about COVID-19 on a five-point Likert-type scale from "not at all worried" to "extremely worried".

Participants were asked approximately what percentage of people of around the same age they thought were "fully following the UK Government's recommendations to stay at home". Participants reported whether they thought the current lockdown had made their physical health better or worse. Possible answers were "a lot better", "a little better", "no difference", "a little worse", and "a lot worse".

Participants were asked if they had helped, or received help from, someone outside their household in the past seven days (yes / no).

Participants rated fourteen perception statements on a five-point Likert scale from "strongly disagree" to "strongly agree". Statements included perceived severity of COVID-19, perceived effectiveness of Government measures, perceived likelihood of catching and spreading COVID-19, perceived costs of following Government measures, fear of losing touch with friends and relatives, social pressure from friends and family to follow Government measures, perceived legal consequences from not following Government measures, and positive consequences of the lockdown.

\section{Personal and clinical characteristics}

We asked participants to report their age, gender, employment status, highest educational or professional qualification, and their marital status. We also asked participants whether there 
was a child in their household, whether they or someone else in their household received a letter from the NHS telling them they were extremely clinically vulnerable to COVID-19 (proxy for chronic illness), and whether they lived alone. Participants were asked for their postcode to determine indices of multiple deprivation and whether they lived in an urban or rural area. We also collected social grade.

Participants were asked if their primary home had access to any outdoor space, and whether they were pet owners.

\section{Ethics}

Ethical approval for this study was granted by the King's College London Research Ethics Committee (reference: LRS-19/20-18687).

\section{Patient and public involvement}

Due to the rapid nature of this research, the public was not involved in the development of the survey materials.

\section{Power}

A sample size of 2,240 allows a $95 \%$ confidence interval of plus or minus $2 \%$ for the prevalence estimate for each survey item.

\section{Analysis}

Recoding variables

GAD-2 scores of three or over indicated probable anxiety. ${ }^{16}$ PHQ-2 scores of three or over indicated probable depression. ${ }^{17}$ We were unable to calculate a score for anxiety or depression for people who skipped, or answered "don't know" or "prefer not to say", to one or both questions for these scales (GAD-2: $6.0 \%$ of the sample, $n=134$. PHQ-2: $5.4 \%$ of the sample, $\mathrm{n}=122$ ).

Presence of COVID-19 symptoms in the household was recoded into a binary variable. We defined presence of COVID-19 symptoms as reporting having cough or high temperature / fever oneself in the last seven days, or reporting that a household member had a cough or high temperature / fever in the last fourteen days.

We recoded whether participants reported self-isolating into a binary variable (not selfisolating, self-isolating). 
We calculated the total number of outings made in the last seven days by summing the number of times people reported going out (shopping for groceries, toiletries or medicine, shopping for other items, exercising, for a medical purpose, going to work, helping someone else, meeting friends or family). We grouped participants who reported going out more than twenty times in the past seven days $(n=54,2.4 \%)$.

For all variables, unless stated otherwise, we coded answers of "don't know" or "prefer not to say" as missing data.

Analyses

We ran a series of logistic regressions investigating univariable associations between personal and clinical factors, psychological and situational factors and anxiety and depression. We ran a second set of logistic regressions controlling for personal and clinical characteristics.

We ran a series of linear regressions investigating univariable associations between personal and clinical factors, psychological and situational factors and self-rated general health. We ran a second set of linear regressions controlling for personal and clinical characteristics. For these regressions, we used the total proportion of the variance explained by individual psychological and situational factors, in addition to personal and clinical characteristics, and statistical significance of individual regression coefficients to determine which factors were most strongly associated.

Data were weighted to increase their representativeness of the UK population.

Sensitivity analyses

As we ran many analyses on each outcome ( $n=39$ for anxiety and depression, $n=40$ for selfreported general health), we applied a Bonferroni correction to our results ( $p \leq \cdot 001)$. Analyses reaching this significance level are marked by a double asterisk $(* *)$ in the tables.

\section{RESULTS}

Only results of adjusted analyses are reported narratively. Results of unadjusted analyses are reported in the tables.

\section{Anxiety}

Data were available to calculate anxiety scores for 2,089 participants. $21.9 \%(\mathrm{n}=458,95 \% \mathrm{CI}$ [20.1\% to $23.7 \%$ ]) reported probable anxiety (scored three or over on GAD-2). 
Anxiety was associated with being female, younger, not working, and living in a more deprived area (Table 1).

Anxiety was also associated with: the presence of depression; feeling that the current lockdown had made your mental health worse; presence of COVID-19 symptoms in the household; greater worry about COVID-19; having received help from someone outside your household because of COVID-19 in the last week; having more conflict in your household because of the lockdown; reporting that you were currently self-isolating; not enjoying spending more time at home during the lockdown; believing that following Government advice would impact your finances negatively and cause you to lose touch with friends and relatives; greater perceived severity of COVID-19 for your family's wellbeing; lower perceived sense of community with people in your neighbourhood; lower perceived effectiveness of Government measures; and thinking that fewer people of your age in the UK were following the UK Government's recommendations to stay at home (lower perceived social norms; Table 2). 
Table 1. Associations between personal and clinical characteristics and anxiety and depression.

\begin{tabular}{|c|c|c|c|c|c|c|c|c|c|}
\hline \multirow{2}{*}{$\begin{array}{l}\text { Participant } \\
\text { characteristics }\end{array}$} & \multirow[t]{2}{*}{ Level } & \multicolumn{8}{|c|}{ Psychological distress } \\
\hline & & $\begin{array}{l}\text { No probable } \\
\text { anxiety } \\
\mathrm{n}=1632, \mathrm{n} \\
(\%)\end{array}$ & $\begin{array}{l}\text { Probable } \\
\text { anxiety } \\
\mathrm{n}=458, \mathrm{n} \\
(\%)\end{array}$ & $\begin{array}{l}\text { Odds ratio } \\
(95 \% \mathrm{CI})\end{array}$ & $\begin{array}{l}\text { Adjusted } \\
\text { odds ratio } \\
(95 \% \text { CI }) \dagger\end{array}$ & $\begin{array}{l}\text { No probable } \\
\text { depression } \\
n=1610, n(\%)\end{array}$ & $\begin{array}{l}\text { Probable } \\
\text { depression } \\
\mathrm{n}=494, \mathrm{n}(\%)\end{array}$ & $\begin{array}{l}\text { Odds ratio } \\
(95 \% \mathrm{CI})\end{array}$ & $\begin{array}{l}\text { Adjusted } \\
\text { odds ratio } \\
(95 \% \text { CI }) \dagger\end{array}$ \\
\hline \multirow[t]{2}{*}{ Gender } & Male & $829(82 \cdot 8)$ & $172(17 \cdot 2)$ & Reference & Reference & $796(78 \cdot 0)$ & $225(22 \cdot 0)$ & Reference & Reference \\
\hline & Female & $803(73 \cdot 8)$ & $285(26 \cdot 2)$ & $\begin{array}{l}1.71(1.38 \\
\text { to } 2 \cdot 12)^{* *}\end{array}$ & $\begin{array}{l}2.00(1.57 \text { to } \\
2.55)^{* *}\end{array}$ & $814(75 \cdot 2)$ & $269(24 \cdot 8)$ & $\begin{array}{l}1 \cdot 17(0 \cdot 96 \\
\text { to } 1 \cdot 43)\end{array}$ & $\begin{array}{l}1.23(0.98 \text { to } \\
1.54)\end{array}$ \\
\hline \multirow[t]{5}{*}{ Age } & 18 to 24 years & $126(58 \cdot 6)$ & $89(41.4)$ & Reference & Reference & $130(58 \cdot 6)$ & $92(41.4)$ & Reference & Reference \\
\hline & 25 to 34 years & $219(72 \cdot 3)$ & $84(27 \cdot 7)$ & $\begin{array}{l}0.55(0 \cdot 38 \\
\text { to } 0.79)^{* * *}\end{array}$ & $\begin{array}{l}0.50(0.31 \text { to } \\
0.79) *\end{array}$ & $213(71 \cdot 2)$ & $86(28.8)$ & $\begin{array}{l}0.57(0 \cdot 40 \\
\text { to } 0.83)^{*}\end{array}$ & $\begin{array}{l}0.66(0.41 \text { to } \\
1.04)\end{array}$ \\
\hline & 35 to 44 years & $298(79 \cdot 3)$ & $78(20 \cdot 7)$ & $\begin{array}{l}0 \cdot 37(0 \cdot 26 \\
\text { to } 0 \cdot 54)^{* * *}\end{array}$ & $\begin{array}{l}0.30(0.19 \text { to } \\
0.48)^{* *}\end{array}$ & $278(74.9)$ & $93(25 \cdot 1)$ & $\begin{array}{l}0.48(0.33 \\
\text { to } 0.68)^{* *}\end{array}$ & $\begin{array}{l}0.55(0.35 \text { to } \\
0.87)^{*}\end{array}$ \\
\hline & 45 to 54 years & $274(77 \cdot 0)$ & $82(23 \cdot 0)$ & $\begin{array}{l}0.42(0 \cdot 29 \\
\text { to } 0 \cdot 61)^{* *}\end{array}$ & $\begin{array}{l}0.37(0.23 \text { to } \\
0.59)^{* *}\end{array}$ & $268(75 \cdot 3)$ & $88(24 \cdot 7)$ & $\begin{array}{l}0.47(0 \cdot 33 \\
\text { to } 0 \cdot 67)^{* *}\end{array}$ & $\begin{array}{l}0.56(0.36 \text { to } \\
0 \cdot 90)^{*}\end{array}$ \\
\hline & 55 years and over & $715(85 \cdot 1)$ & $125(14 \cdot 9)$ & $\begin{array}{l}0.25(0 \cdot 18 \\
\text { to } 0 \cdot 35)^{* *}\end{array}$ & $\begin{array}{l}0.21(0.13 \text { to } \\
0.33) * *\end{array}$ & $722(84 \cdot 3)$ & $134(15 \cdot 7)$ & $\begin{array}{l}0 \cdot 26(0 \cdot 19 \\
\text { to } 0 \cdot 36)^{* *}\end{array}$ & $\begin{array}{l}0 \cdot 26(0 \cdot 17 \text { to } \\
0 \cdot 41)^{* *}\end{array}$ \\
\hline \multirow{2}{*}{$\begin{array}{l}\text { Child in the } \\
\text { household }\end{array}$} & None & $1201(79 \cdot 5)$ & $309(20 \cdot 5)$ & Reference & Reference & $1181(77 \cdot 6)$ & $341(22 \cdot 4)$ & Reference & Reference \\
\hline & Present & $415(75 \cdot 5)$ & $135(24 \cdot 5)$ & $\begin{array}{l}1.27(1.01 \\
\text { to } 1.60)^{*}\end{array}$ & $\begin{array}{l}1 \cdot 01(0 \cdot 76 \text { to } \\
1 \cdot 35)\end{array}$ & $411(74 \cdot 6)$ & $140(25 \cdot 4)$ & $\begin{array}{l}1 \cdot 18(0 \cdot 94 \\
\text { to } 1 \cdot 48)\end{array}$ & $\begin{array}{l}1.06(0.80 \text { to } \\
1.40)\end{array}$ \\
\hline \multirow{2}{*}{$\begin{array}{l}\text { Clinically extremely } \\
\text { vulnerable (self) }\end{array}$} & No & $1491(78 \cdot 5)$ & $409(21.5)$ & Reference & Reference & $1481(77 \cdot 7)$ & $424(22 \cdot 3)$ & Reference & Reference \\
\hline & Yes & $99(77 \cdot 3)$ & $29(22 \cdot 7)$ & $\begin{array}{l}1.07(0 \cdot 70 \\
\text { to } 1 \cdot 64)\end{array}$ & $\begin{array}{l}1.18(0.74 \text { to } \\
1.9)\end{array}$ & $92(70 \cdot 2)$ & $39(29 \cdot 8)$ & $\begin{array}{l}1.49(1 \cdot 01 \\
\text { to } 2 \cdot 20)^{*}\end{array}$ & $\begin{array}{l}1.70(1.11 \text { to } \\
2.59)^{*}\end{array}$ \\
\hline \multirow[t]{2}{*}{ Employment status } & Not working & $741(77 \cdot 0)$ & $221(23 \cdot 0)$ & Reference & Reference & $733(75 \cdot 4)$ & $239(24 \cdot 6)$ & Reference & Reference \\
\hline & Working & $891(79 \cdot 1)$ & $236(20 \cdot 9)$ & $\begin{array}{l}0.89(0 \cdot 72 \\
\text { to } 1.09)\end{array}$ & $\begin{array}{l}0.71(0.54 \text { to } \\
0.92)^{*}\end{array}$ & $877(77 \cdot 5)$ & $254(22 \cdot 5)$ & $\begin{array}{l}0.89(0.73 \\
\text { to } 1.09)\end{array}$ & $\begin{array}{l}0.65(0.50 \text { to } \\
0 \cdot 85)^{* *}\end{array}$ \\
\hline \multirow{2}{*}{$\begin{array}{l}\text { Highest educational } \\
\text { or professional } \\
\text { qualification }\end{array}$} & $\begin{array}{l}\text { GCSE/vocational/A-level/No formal } \\
\text { qualifications }\end{array}$ & $755(77 \cdot 0)$ & $225(23 \cdot 0)$ & Reference & Reference & $748(75 \cdot 5)$ & $243(24 \cdot 5)$ & Reference & Reference \\
\hline & $\begin{array}{l}\text { Degree or higher (Bachelors, } \\
\text { Masters, PhD) }\end{array}$ & $856(79 \cdot 7)$ & $218(20 \cdot 3)$ & $\begin{array}{l}0.86(0 \cdot 69 \\
\text { to } 1 \cdot 06)\end{array}$ & $\begin{array}{l}0.90(0.70 \text { to } \\
1.14)\end{array}$ & $841(78 \cdot 1)$ & $236(21 \cdot 9)$ & $\begin{array}{l}0.86(0 \cdot 70 \\
\text { to } 1 \cdot 06)\end{array}$ & $\begin{array}{l}1.00(0.79 \text { to } \\
1.27)\end{array}$ \\
\hline IMD decile & $\begin{array}{l}\text { Range } 1 \text { (most deprived) to } 10 \text { (least } \\
\text { deprived) }\end{array}$ & $\begin{array}{l}\mathrm{N}=1632 \\
\mathrm{M}=6 \cdot 06 \\
\mathrm{SD}=2 \cdot 75\end{array}$ & $\begin{array}{l}\mathrm{N}=458 \\
\mathrm{M}=5 \cdot 31 \\
\mathrm{SD}=2 \cdot 93\end{array}$ & $\begin{array}{l}0.91(0 \cdot 88 \\
\text { to } 0.94)^{* *}\end{array}$ & $\begin{array}{l}0.92(0 \cdot 88 \text { to } \\
0 \cdot 96)^{* *}\end{array}$ & $\begin{array}{l}\mathrm{N}=1610 \\
\mathrm{M}=6 \cdot 11 \\
\mathrm{SD}=2 \cdot 93\end{array}$ & $\begin{array}{l}\mathrm{N}=494 \\
\mathrm{M}=5 \cdot 32 \\
\mathrm{SD}=2 \cdot 92\end{array}$ & $\begin{array}{l}0.90(0 \cdot 87 \\
\text { to } 0 \cdot 94)^{* *}\end{array}$ & $\begin{array}{l}0.93(0.89 \text { to } \\
0.97)^{* *}\end{array}$ \\
\hline \multirow[t]{2}{*}{ Social grade } & $\mathrm{ABC1}$ & $950(79 \cdot 4)$ & $247(20 \cdot 6)$ & Reference & Reference & $940(78 \cdot 0)$ & $265(22 \cdot 0)$ & Reference & Reference \\
\hline & $\mathrm{C} 2 \mathrm{DE}$ & $682(76 \cdot 4)$ & $211(23 \cdot 6)$ & $\begin{array}{l}1.19(0.97 \\
\text { to } 1.47)\end{array}$ & $\begin{array}{l}1 \cdot 04(0.81 \text { to } \\
1 \cdot 33)\end{array}$ & $670(74 \cdot 5)$ & $229(25 \cdot 5)$ & $\begin{array}{l}1.22(0.99 \\
\text { to } 1.49)\end{array}$ & $\begin{array}{l}1.12(0.88 \text { to } \\
1.42)\end{array}$ \\
\hline \multirow[t]{2}{*}{ Urban/rural } & Urban & $1233(76 \cdot 7)$ & $374(23 \cdot 3)$ & Reference & Reference & $1206(74 \cdot 7)$ & $408(25 \cdot 3)$ & Reference & Reference \\
\hline & Rural & $360(84 \cdot 1)$ & $68(15 \cdot 9)$ & $\begin{array}{l}0.62(0 \cdot 47 \\
\text { to } 0 \cdot 82)^{* *}\end{array}$ & $\begin{array}{l}0.79(0.57 \text { to } \\
1.08)\end{array}$ & $361(83 \cdot 2)$ & $73(16 \cdot 8)$ & $\begin{array}{l}0.60(0.45 \\
\text { to } 0.79) * *\end{array}$ & $\begin{array}{l}0.75(0.55 \text { to } \\
1.02)\end{array}$ \\
\hline
\end{tabular}




\begin{tabular}{|c|c|c|c|c|c|c|c|c|c|}
\hline Living alone & $\begin{array}{l}\text { Yes } \\
\text { No }\end{array}$ & $\begin{array}{l}319(79 \cdot 9) \\
1313(77 \cdot 8)\end{array}$ & $\begin{array}{l}80(20 \cdot 1) \\
375(22 \cdot 2)\end{array}$ & $\begin{array}{l}\text { Reference } \\
1 \cdot 14(0 \cdot 87 \\
\text { to } 1.50)\end{array}$ & $\begin{array}{l}\text { Reference } \\
1.04(0.70 \text { to } \\
1.53)\end{array}$ & $\begin{array}{l}294(72 \cdot 1) \\
1316(77 \cdot 6)\end{array}$ & $\begin{array}{l}114(27 \cdot 9) \\
379(22 \cdot 4)\end{array}$ & $\begin{array}{l}\text { Reference } \\
0.74(0.58 \\
\text { to } 0.95)^{*}\end{array}$ & $\begin{array}{l}\text { Reference } \\
0.73(0.51 \text { to } \\
1.04)\end{array}$ \\
\hline \multirow[t]{2}{*}{ Marital status } & $\begin{array}{l}\text { Married/civil partnership/living as } \\
\text { married }\end{array}$ & $996(80 \cdot 8)$ & $236(19 \cdot 2)$ & Reference & Reference & $1003(81 \cdot 6)$ & $226(18 \cdot 4)$ & Reference & Reference \\
\hline & $\begin{array}{l}\text { Separated/divorced/widowed/never } \\
\text { married }\end{array}$ & $626(74 \cdot 1)$ & $219(25 \cdot 9)$ & $\begin{array}{l}1.48(1.20 \\
\text { to } 1.82)^{* *}\end{array}$ & $\begin{array}{l}1.07(0.78 \text { to } \\
1.48)\end{array}$ & $594(69 \cdot 2)$ & $264(30 \cdot 8)$ & $\begin{array}{l}1.97(1.61 \\
\text { to } 2.42)^{* *}\end{array}$ & $\begin{array}{l}1.31(0.96 \text { to } \\
1.80)\end{array}$ \\
\hline $\begin{array}{l}\text { Clinically extremely } \\
\text { vulnerable } \\
\text { (household member) } \\
+\end{array}$ & $\begin{array}{l}\text { No } \\
\text { Yes }\end{array}$ & $\begin{array}{l}1171(78 \cdot 4) \\
111(75 \cdot 5)\end{array}$ & $\begin{array}{l}323(21 \cdot 6) \\
36(24 \cdot 5)\end{array}$ & $\begin{array}{l}\text { Reference } \\
1 \cdot 18(0 \cdot 79 \\
\text { to } 1.75)\end{array}$ & $\begin{array}{l}\text { Reference } \\
1.01(0.64 \text { to } \\
1.59)\end{array}$ & $\begin{array}{l}1165(78 \cdot 2) \\
124(80 \cdot 0)\end{array}$ & $\begin{array}{l}324(21 \cdot 8) \\
31(20 \cdot 0)\end{array}$ & $\begin{array}{l}\text { Reference } \\
0.89(0.59 \\
\text { to } 1.34)\end{array}$ & $\begin{array}{l}\text { Reference } \\
0 \cdot 61(0 \cdot 37 \text { to } \\
1 \cdot 00)^{*}\end{array}$ \\
\hline $\begin{array}{l}\text { Home includes } \\
\text { access to outside } \\
\text { space }\end{array}$ & $\begin{array}{l}\text { No } \\
\text { Yes }\end{array}$ & $\begin{array}{l}128(74 \cdot 0) \\
1504(78 \cdot 5)\end{array}$ & $\begin{array}{l}45(26 \cdot 0) \\
412(21 \cdot 5)\end{array}$ & $\begin{array}{l}\text { Reference } \\
0.78(0.54 \\
\text { to } 1 \cdot 11)\end{array}$ & $\begin{array}{l}\text { Reference } \\
1.03(0.66 \text { to } \\
1.60)\end{array}$ & $\begin{array}{l}113(68 \cdot 5) \\
1497(77 \cdot 2)\end{array}$ & $\begin{array}{l}52(31 \cdot 5) \\
441(22 \cdot 8)\end{array}$ & $\begin{array}{l}\text { Reference } \\
0.64(0.45 \\
\text { to } 0.90)^{*}\end{array}$ & $\begin{array}{l}\text { Reference } \\
0.93(0.61 \text { to } \\
1.41)\end{array}$ \\
\hline Pet ownership & $\begin{array}{l}\text { No } \\
\text { Yes }\end{array}$ & $\begin{array}{l}878(78 \cdot 5) \\
754(77 \cdot 7)\end{array}$ & $\begin{array}{l}241(21 \cdot 5) \\
216(22 \cdot 3)\end{array}$ & $\begin{array}{l}\text { Reference } \\
1.04(0.85 \\
\text { to } 1.29)\end{array}$ & $\begin{array}{l}\text { Reference } \\
0.95(0.75 \text { to } \\
1.22)\end{array}$ & $\begin{array}{l}878(78 \cdot 0) \\
731(74 \cdot 7)\end{array}$ & $\begin{array}{l}247(22 \cdot 0) \\
247(25 \cdot 3)\end{array}$ & $\begin{array}{l}\text { Reference } \\
1.20(0.98 \\
\text { to } 1.47)\end{array}$ & $\begin{array}{l}\text { Reference } \\
1 \cdot 16(0.91 \text { to } \\
1.46)\end{array}$ \\
\hline
\end{tabular}

$* p \leq \cdot 05$

$* * p \leq \cdot 001$

† Adjusting for gender, age, having a child in the household, being extremely clinically vulnerable oneself, employment status, highest level of education or professional qualification, indices of multiple deprivation, social grade, living in a rural or urban area, living alone, marital status, and region.

$\ddagger$ Adjusted analyses for this variable did not control for living alone, as by definition all participants asked this question lived in a household with someone else. 
Table 2. Associations between psychological and situational factors and anxiety and depression.

\begin{tabular}{|c|c|c|c|c|c|c|c|c|c|}
\hline \multirow[t]{2}{*}{ Participant characteristics } & \multirow[t]{2}{*}{ Level } & \multicolumn{8}{|c|}{ Psychological distress } \\
\hline & & $\begin{array}{l}\text { No probable } \\
\text { anxiety } \\
\mathrm{n}=1632, \mathrm{n}(\%)\end{array}$ & $\begin{array}{l}\text { Probable } \\
\text { anxiety } \\
n=458, n(\%)\end{array}$ & $\begin{array}{l}\text { Odds ratio } \\
(95 \% \mathrm{CI})\end{array}$ & $\begin{array}{l}\text { Adjusted } \\
\text { odds ratio } \\
(95 \% \mathrm{CI}) \dagger\end{array}$ & $\begin{array}{l}\text { No probable } \\
\text { depression } \\
\mathrm{n}=1610, \mathrm{n}(\%)\end{array}$ & $\begin{array}{l}\text { Probable } \\
\text { depression } \\
\mathrm{n}=494, \mathrm{n}(\%)\end{array}$ & $\begin{array}{l}\text { Odds ratio } \\
(95 \% \mathrm{CI})\end{array}$ & $\begin{array}{l}\text { Adjusted } \\
\text { odds ratio } \\
(95 \% \text { CI }) \dagger\end{array}$ \\
\hline Probable anxiety & $\begin{array}{l}\text { None } \\
\text { Present }\end{array}$ & 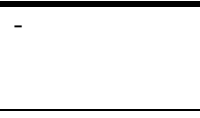 & 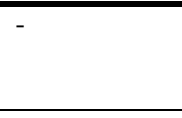 & - & - & $\begin{array}{l}1441(90 \cdot 2) \\
124(28 \cdot 4)\end{array}$ & $\begin{array}{l}157(9 \cdot 8) \\
312(71 \cdot 6)\end{array}$ & $\begin{array}{l}\text { Reference } \\
23 \cdot 12(17 \cdot 73 \\
\text { to } 30 \cdot 14)^{* *} \\
\end{array}$ & $\begin{array}{l}\text { Reference } \\
22 \cdot 00(16 \cdot 25 \\
\text { to } 29 \cdot 80)^{* * *}\end{array}$ \\
\hline Probable depression & $\begin{array}{l}\text { None } \\
\text { Present }\end{array}$ & $\begin{array}{l}1441(92 \cdot 1) \\
157(33 \cdot 5)\end{array}$ & $\begin{array}{l}124(7 \cdot 9) \\
312(66 \cdot 5)\end{array}$ & $\begin{array}{l}\text { Reference } \\
23 \cdot 12(17 \cdot 73 \\
\text { to } 30 \cdot 14)^{* *}\end{array}$ & $\begin{array}{l}\text { Reference } \\
21 \cdot 78(16 \cdot 09 \\
\text { to } 29 \cdot 49)^{* *}\end{array}$ & - & - & - & - \\
\hline \multirow[t]{2}{*}{$\begin{array}{l}\text { Had, or currently have, } \\
\text { COVID-19 }\end{array}$} & $\begin{array}{l}\text { Think have not had } \\
\text { COVID-19 and do } \\
\text { not have it now }\end{array}$ & $1280(79.9)$ & $322(20 \cdot 1)$ & Reference & Reference & $1272(78 \cdot 6)$ & $347(21 \cdot 4)$ & Reference & Reference \\
\hline & $\begin{array}{l}\text { Think have had } \\
\text { COVID-19 or have it } \\
\text { now }\end{array}$ & $156(73 \cdot 9)$ & $55(26 \cdot 1)$ & $\begin{array}{l}1.40(1.01 \text { to } \\
1.95)^{*}\end{array}$ & $\begin{array}{l}1.33(0.91 \text { to } \\
1.94)\end{array}$ & $15(72 \cdot 2)$ & $58(27 \cdot 8)$ & $\begin{array}{l}1.41(1.02 \text { to } \\
1.95)^{*}\end{array}$ & $\begin{array}{l}1.34(0.93 \text { to } \\
1.93)\end{array}$ \\
\hline \multirow[t]{2}{*}{$\begin{array}{l}\text { Symptoms of COVID-19 in } \\
\text { the household }\end{array}$} & $\begin{array}{l}\text { No symptoms } \\
\text { present }\end{array}$ & $1448(79 \cdot 6)$ & $370(20 \cdot 4)$ & Reference & Reference & $144(77 \cdot 4)$ & $412(22 \cdot 6)$ & Reference & Reference \\
\hline & Symptoms present & $140(70 \cdot 0)$ & $60(30 \cdot 0)$ & $\begin{array}{l}1.67(1.21 \text { to } \\
2 \cdot 30)^{*}\end{array}$ & $\begin{array}{l}1.79(1.24 \text { to } \\
2 \cdot 57)^{*}\end{array}$ & $136(69 \cdot 0)$ & $61(31 \cdot 0)$ & $\begin{array}{l}1.54(1.12 \text { to } \\
2.13)^{*}\end{array}$ & $\begin{array}{l}1.59(1.10 \text { to } \\
2 \cdot 28)^{*}\end{array}$ \\
\hline Self-isolating & $\begin{array}{l}\text { Not self-isolating } \\
\text { Self-isolating }\end{array}$ & $\begin{array}{l}1240(78 \cdot 0) \\
384(79 \cdot 3)\end{array}$ & $\begin{array}{l}350(22 \cdot 0) \\
100(20 \cdot 7)\end{array}$ & $\begin{array}{l}\text { Reference } \\
1.31(1.03 \text { to } \\
1.65)^{*}\end{array}$ & $\begin{array}{l}\text { Reference } \\
1.43(1.07 \text { to } \\
1.91)^{*}\end{array}$ & $\begin{array}{l}1244(77 \cdot 6) \\
366(73 \cdot 2)\end{array}$ & $\begin{array}{l}359(22 \cdot 4) \\
134(26 \cdot 8)\end{array}$ & $\begin{array}{l}\text { Reference } \\
1.27(1.01 \text { to } \\
1.60)^{*}\end{array}$ & $\begin{array}{l}\text { Reference } \\
1.20(0.91 \text { to } \\
1.60)\end{array}$ \\
\hline $\begin{array}{l}\text { Total out of home activity in } \\
\text { past week }\end{array}$ & Range 0 to $20+$ & $\begin{array}{l}\mathrm{N}=1632, \\
\mathrm{M}=6 \cdot 79 \\
\mathrm{SD}=5 \cdot 12\end{array}$ & $\begin{array}{l}\mathrm{N}=458 \\
\mathrm{M}=6 \cdot 27 \\
\mathrm{SD}=5 \cdot 38\end{array}$ & $\begin{array}{l}0.98(0.96 \text { to } \\
1.00)\end{array}$ & $\begin{array}{l}1(0.97 \text { to } \\
1.02)\end{array}$ & $\begin{array}{l}\mathrm{N}=1610, \\
\mathrm{M}=6 \cdot 78 \\
\mathrm{SD}=5 \cdot 06\end{array}$ & $\begin{array}{l}\mathrm{N}=494 \\
\mathrm{M}=6 \cdot 48 \\
\mathrm{SD}=5 \cdot 58\end{array}$ & $\begin{array}{l}0.99(0.97 \text { to } \\
1.01)\end{array}$ & $\begin{array}{l}1.00(0.98 \text { to } \\
1.03)\end{array}$ \\
\hline Worry about COVID-19 & $\begin{array}{l}5 \text {-point scale, } 1=\text { not } \\
\text { at all worried to } \\
5=\text { extremely worried }\end{array}$ & $\begin{array}{l}\mathrm{N}=1627 \\
\mathrm{M}=3 \cdot 19 \\
\mathrm{SD}=0 \cdot 99\end{array}$ & $\begin{array}{l}\mathrm{N}=455 \\
\mathrm{M}=3 \cdot 64 \\
\mathrm{SD}=1 \cdot 03\end{array}$ & $\begin{array}{l}1.57(1.40 \text { to } \\
1.75)^{* *}\end{array}$ & $\begin{array}{l}1.71(1.50 \text { to } \\
1.95)^{* *}\end{array}$ & $\begin{array}{l}\mathrm{N}=1606 \\
\mathrm{M}=3 \cdot 26 \\
\mathrm{SD}=0 \cdot 98\end{array}$ & $\begin{array}{l}\mathrm{N}=491 \\
\mathrm{M}=3.37 \\
\mathrm{SD}=1.08\end{array}$ & $\begin{array}{l}1.11(1.01 \text { to } \\
1.23)^{*}\end{array}$ & $\begin{array}{l}1.16(1.03 \text { to } \\
1.31)^{*}\end{array}$ \\
\hline Perceived social norms & $\begin{array}{l}\text { Percentage (range 0- } \\
100)\end{array}$ & $\begin{array}{l}\mathrm{N}=1495 \\
\mathrm{M}=73.67 \\
\mathrm{SD}=15.79\end{array}$ & $\begin{array}{l}\mathrm{N}=391, \\
\mathrm{M}=66 \cdot 96 \\
\mathrm{SD}=18 \cdot 57\end{array}$ & $\begin{array}{l}0.98(0 \cdot 97 \text { to } \\
0 \cdot 98)^{* *}\end{array}$ & $\begin{array}{l}0.98(0 \cdot 98 \text { to } \\
0.99)^{* *}\end{array}$ & $\begin{array}{l}\mathrm{N}=1481 \\
\mathrm{M}=73 \cdot 56 \\
\mathrm{SD}=15 \cdot 86\end{array}$ & $\begin{array}{l}\mathrm{N}=417 \\
\mathrm{M}=67 \cdot 13 \\
\mathrm{SD}=19 \cdot 15\end{array}$ & $\begin{array}{l}0.98(0 \cdot 97 \text { to } \\
0.99)^{* *}\end{array}$ & $\begin{array}{l}0.98(0.98 \text { to } \\
0.99)^{* *}\end{array}$ \\
\hline $\begin{array}{l}\text { Perceptions about impact on } \\
\text { mental health }\end{array}$ & $\begin{array}{l}5 \text {-point scale, } 1=\text { a lot } \\
\text { better to } 5=\text { a lot } \\
\text { worse }\end{array}$ & $\begin{array}{l}\mathrm{N}=1621 \\
\mathrm{M}=3 \cdot 26 \\
\mathrm{SD}=0 \cdot 82\end{array}$ & $\begin{array}{l}\mathrm{N}=447 \\
\mathrm{M}=4 \cdot 14 \\
\mathrm{SD}=0 \cdot 92\end{array}$ & $\begin{array}{l}3 \cdot 54(3 \cdot 05 \text { to } \\
4 \cdot 10)^{* *}\end{array}$ & $\begin{array}{l}3 \cdot 50(2 \cdot 96 \text { to } \\
4 \cdot 14)^{* *}\end{array}$ & $\begin{array}{l}\mathrm{N}=1601 \\
\mathrm{M}=3 \cdot 24 \\
\mathrm{SD}=0 \cdot 80\end{array}$ & $\begin{array}{l}\mathrm{N}=483 \\
\mathrm{M}=4 \cdot 21 \\
\mathrm{SD}=0 \cdot 87\end{array}$ & $\begin{array}{l}4 \cdot 53(3 \cdot 87 \text { to } \\
5 \cdot 30)^{* *}\end{array}$ & $\begin{array}{l}4.56(3.82 \text { to } \\
5.45)^{* *}\end{array}$ \\
\hline $\begin{array}{l}\text { Perceptions about impact on } \\
\text { physical health }\end{array}$ & $\begin{array}{l}5 \text {-point scale, } 1=a \text { lot } \\
\text { better to } 5=a \text { lot } \\
\text { worse }\end{array}$ & $\begin{array}{l}\mathrm{N}=1627 \\
\mathrm{M}=3 \cdot 10 \\
\mathrm{SD}=0 \cdot 91\end{array}$ & $\begin{array}{l}\mathrm{N}=450 \\
\mathrm{M}=3 \cdot 37 \\
\mathrm{SD}=1 \cdot 00\end{array}$ & $\begin{array}{l}1.37(1.22 \text { to } \\
1.53)^{* *}\end{array}$ & $\begin{array}{l}1.39(1.23 \text { to } \\
1.59)^{* *}\end{array}$ & $\begin{array}{l}\mathrm{N}=1605 \\
\mathrm{M}=3 \cdot 08 \\
\mathrm{SD}=0 \cdot 90\end{array}$ & $\begin{array}{l}\mathrm{N}=487 \\
\mathrm{M}=3 \cdot 48 \\
\mathrm{SD}=0 \cdot 98\end{array}$ & $\begin{array}{l}1.63(1.45 \text { to } \\
1.83) * *\end{array}$ & $\begin{array}{l}1.66(1.46 \text { to } \\
1.89)^{* *}\end{array}$ \\
\hline Helped someone outside & No & $1240(78 \cdot 0)$ & $350(22 \cdot 0)$ & Reference & Reference & $1215(75 \cdot 9)$ & $385(24 \cdot 1)$ & Reference & Reference \\
\hline
\end{tabular}




\begin{tabular}{|c|c|c|c|c|c|c|c|c|c|}
\hline household & Yes & $384(79 \cdot 3)$ & $100(20 \cdot 7)$ & $\begin{array}{l}0.93(0.72 \text { to } \\
1.19)\end{array}$ & $\begin{array}{l}1.04(0.79 \text { to } \\
1.37)\end{array}$ & $388(79 \cdot 0)$ & $103(21 \cdot 0)$ & $\begin{array}{l}0.84(0.66 \text { to } \\
1.08)\end{array}$ & $\begin{array}{l}0.96(0.73 \text { to } \\
1.25)\end{array}$ \\
\hline $\begin{array}{l}\text { Received help from someone } \\
\text { outside household }\end{array}$ & $\begin{array}{l}\text { No } \\
\text { Yes }\end{array}$ & $\begin{array}{l}1422(79 \cdot 0) \\
202(73 \cdot 7)\end{array}$ & $\begin{array}{l}378(21 \cdot 0) \\
72(26 \cdot 3)\end{array}$ & $\begin{array}{l}\text { Reference } \\
1.35(1.01 \text { to } \\
1.80)^{*} \\
\end{array}$ & $\begin{array}{l}\text { Reference } \\
1.62(1 \cdot 14 \text { to } \\
2 \cdot 30)^{*} \\
\end{array}$ & $\begin{array}{l}1400(77 \cdot 3) \\
203(72 \cdot 2)\end{array}$ & $\begin{array}{l}410(22 \cdot 7) \\
78(27 \cdot 8)\end{array}$ & $\begin{array}{l}\text { Reference } \\
1.31(0.99 \text { to } \\
1.75) \\
\end{array}$ & $\begin{array}{l}\text { Reference } \\
1.43(1.02 \text { to } \\
2.01) \\
\end{array}$ \\
\hline $\begin{array}{l}\text { If I completely follow the } \\
\text { Government's advice, I will } \\
\text { lose touch with my friends } \\
\text { and relatives }\end{array}$ & $\begin{array}{l}\text { 5-point scale, } \\
1=\text { strongly disagree } \\
\text { to } 5=\text { strongly agree }\end{array}$ & $\begin{array}{l}\mathrm{N}=1620 \\
\mathrm{M}=1 \cdot 97 \\
\mathrm{SD}=1 \cdot 06\end{array}$ & $\begin{array}{l}\mathrm{N}=452 \\
\mathrm{M}=2 \cdot 33 \\
\mathrm{SD}=1 \cdot 24\end{array}$ & $\begin{array}{l}1.31(1.20 \text { to } \\
1.43)^{* *}\end{array}$ & $\begin{array}{l}1.33(1.20 \text { to } \\
1.48)^{* *}\end{array}$ & $\begin{array}{l}\mathrm{N}=1601 \\
\mathrm{M}=1 \cdot 93 \\
\mathrm{SD}=1 \cdot 03\end{array}$ & $\begin{array}{l}\mathrm{N}=486 \\
\mathrm{M}=2 \cdot 47 \\
\mathrm{SD}=1 \cdot 29\end{array}$ & $\begin{array}{l}1.50(1.38 \text { to } \\
1.64)^{* *}\end{array}$ & $\begin{array}{l}1.45(1.31 \text { to } \\
1.60)^{* *}\end{array}$ \\
\hline $\begin{array}{l}\text { My friends or family will } \\
\text { disapprove if I don't follow } \\
\text { the Government's advice }\end{array}$ & $\begin{array}{l}\text { 5-point scale, } \\
1=\text { =strongly disagree } \\
\text { to } 5=\text { strongly agree }\end{array}$ & $\begin{array}{l}\mathrm{N}=1596 \\
\mathrm{M}=4 \cdot 04 \\
\mathrm{SD}=0 \cdot 98\end{array}$ & $\begin{array}{l}\mathrm{N}=446 \\
\mathrm{M}=4 \cdot 01 \\
\mathrm{SD}=0 \cdot 97\end{array}$ & $\begin{array}{l}0.97(0.87 \text { to } \\
1.08)\end{array}$ & $\begin{array}{l}0.98(0.86 \text { to } \\
1 \cdot 10)\end{array}$ & $\begin{array}{l}\mathrm{N}=1581 \\
\mathrm{M}=4 \cdot 06 \\
\mathrm{SD}=0.95\end{array}$ & $\begin{array}{l}\mathrm{N}=477 \\
\mathrm{M}=3.91 \\
\mathrm{SD}=1.06\end{array}$ & $\begin{array}{l}0.86(0.78 \text { to } \\
0.95)^{*}\end{array}$ & $\begin{array}{l}0.87(0.78 \text { to } \\
0.98)^{*}\end{array}$ \\
\hline $\begin{array}{l}\text { If I don't follow the } \\
\text { Government's advice, I could } \\
\text { get in trouble with the police }\end{array}$ & $\begin{array}{l}\text { 5-point scale, } \\
1=\text { strongly disagree } \\
\text { to } 5=\text { strongly agree }\end{array}$ & $\begin{array}{l}\mathrm{N}=1611 \\
\mathrm{M}=3 \cdot 92 \\
\mathrm{SD}=0 \cdot 88\end{array}$ & $\begin{array}{l}\mathrm{N}=450 \\
\mathrm{M}=3 \cdot 99 \\
\mathrm{SD}=0 \cdot 89\end{array}$ & $\begin{array}{l}1.08(0.96 \text { to } \\
1.22)\end{array}$ & $\begin{array}{l}1 \cdot 11(0.97 \text { to } \\
1 \cdot 27)\end{array}$ & $\begin{array}{l}\mathrm{N}=1589 \\
\mathrm{M}=3 \cdot 94 \\
\mathrm{SD}=0 \cdot 88\end{array}$ & $\begin{array}{l}\mathrm{N}=485 \\
\mathrm{M}=3 \cdot 90 \\
\mathrm{SD}=0 \cdot 93\end{array}$ & $\begin{array}{l}0.96(0.86 \text { to } \\
1.08)\end{array}$ & $\begin{array}{l}0.97(0 \cdot 85 \text { to } \\
1 \cdot 10)\end{array}$ \\
\hline $\begin{array}{l}\text { If I follow the Government's } \\
\text { advice, it will help save lives }\end{array}$ & $\begin{array}{l}\text { 5-point scale, } \\
1=\text { strongly disagree } \\
\text { to } 5=\text { strongly agree }\end{array}$ & $\begin{array}{l}\mathrm{N}=1622 \\
\mathrm{M}=4 \cdot 48 \\
\mathrm{SD}=0 \cdot 77\end{array}$ & $\begin{array}{l}\mathrm{N}=455 \\
\mathrm{M}=4 \cdot 34 \\
\mathrm{SD}=0 \cdot 99\end{array}$ & $\begin{array}{l}0.83(0 \cdot 73 \text { to } \\
0.93)^{* *}\end{array}$ & $\begin{array}{l}0.86(0 \cdot 74 \text { to } \\
0.99)^{*}\end{array}$ & $\begin{array}{l}\mathrm{N}=1604 \\
\mathrm{M}=4 \cdot 51 \\
\mathrm{SD}=0 \cdot 74\end{array}$ & $\begin{array}{l}\mathrm{N}=487 \\
\mathrm{M}=4 \cdot 23 \\
\mathrm{SD}=1 \cdot 05\end{array}$ & $\begin{array}{l}0.70(0.63 \text { to } \\
0.78)^{* *}\end{array}$ & $\begin{array}{l}0.76(0.67 \text { to } \\
0.87)^{* *}\end{array}$ \\
\hline $\begin{array}{l}\text { If I follow the Government's } \\
\text { advice, it will help protect the } \\
\text { NHS }\end{array}$ & $\begin{array}{l}\text { 5-point scale, } \\
1=\text { strongly disagree } \\
\text { to } 5=\text { strongly agree }\end{array}$ & $\begin{array}{l}\mathrm{N}=1623 \\
\mathrm{M}=4 \cdot 51 \\
\mathrm{SD}=0.76\end{array}$ & $\begin{array}{l}\mathrm{N}=457 \\
\mathrm{M}=4 \cdot 42 \\
\mathrm{SD}=0 \cdot 89\end{array}$ & $\begin{array}{l}0.87(0 \cdot 77 \text { to } \\
0.98)^{*}\end{array}$ & $\begin{array}{l}0.89(0.77 \text { to } \\
1.03)\end{array}$ & $\begin{array}{l}\mathrm{N}=1601 \\
\mathrm{M}=4 \cdot 54 \\
\mathrm{SD}=0 \cdot 72\end{array}$ & $\begin{array}{l}\mathrm{N}=493 \\
\mathrm{M}=4 \cdot 32 \\
\mathrm{SD}=0 \cdot 97\end{array}$ & $\begin{array}{l}0.73(0 \cdot 64 \text { to } \\
0.82)^{* *}\end{array}$ & $\begin{array}{l}0.77(0.67 \text { to } \\
0 \cdot 88)^{* *}\end{array}$ \\
\hline $\begin{array}{l}\text { If I catch coronavirus, I may } \\
\text { become very ill }\end{array}$ & $\begin{array}{l}\text { 5-point scale, } \\
1=\text { =strongly disagree } \\
\text { to } 5=\text { strongly agree }\end{array}$ & $\begin{array}{l}\mathrm{N}=1598 \\
\mathrm{M}=4 \cdot 33 \\
\mathrm{SD}=0 \cdot 90\end{array}$ & $\begin{array}{l}\mathrm{N}=453 \\
\mathrm{M}=4 \cdot 35 \\
\mathrm{SD}=0 \cdot 92\end{array}$ & $\begin{array}{l}1.03(0 \cdot 91 \text { to } \\
1 \cdot 15)\end{array}$ & $\begin{array}{l}1.13(0.99 \text { to } \\
1.30)\end{array}$ & $\begin{array}{l}\mathrm{N}=1579 \\
\mathrm{M}=4 \cdot 34 \\
\mathrm{SD}=0 \cdot 90\end{array}$ & $\begin{array}{l}\mathrm{N}=485 \\
\mathrm{M}=4 \cdot 28 \\
\mathrm{SD}=0 \cdot 95\end{array}$ & $\begin{array}{l}0.93(0.84 \text { to } \\
1.04)\end{array}$ & $\begin{array}{l}1.04(0.91 \text { to } \\
1 \cdot 18)\end{array}$ \\
\hline $\begin{array}{l}\text { If I catch coronavirus, it will } \\
\text { have a severe impact on my } \\
\text { family's wellbeing }\end{array}$ & $\begin{array}{l}\text { 5-point scale, } \\
1=\text { =strongly disagree } \\
\text { to } 5=\text { strongly agree }\end{array}$ & $\begin{array}{l}\mathrm{N}=1584 \\
\mathrm{M}=4 \cdot 00 \\
\mathrm{SD}=1 \cdot 07\end{array}$ & $\begin{array}{l}\mathrm{N}=448 \\
\mathrm{M}=4 \cdot 11 \\
\mathrm{SD}=1 \cdot 05\end{array}$ & $\begin{array}{l}1.10(1.00 \text { to } \\
1.22)\end{array}$ & $\begin{array}{l}1.20(1.06 \text { to } \\
1.36)^{*}\end{array}$ & $\begin{array}{l}\mathrm{N}=1570 \\
\mathrm{M}=4 \cdot 05 \\
\mathrm{SD}=1 \cdot 03\end{array}$ & $\begin{array}{l}\mathrm{N}=480 \\
\mathrm{M}=3 \cdot 90 \\
\mathrm{SD}=1 \cdot 17\end{array}$ & $\begin{array}{l}0 \cdot 88(0 \cdot 80 \text { to } \\
0.97)^{*}\end{array}$ & $\begin{array}{l}0.95(0.85 \text { to } \\
1.06)\end{array}$ \\
\hline $\begin{array}{l}\text { If I leave home and meet } \\
\text { other people, I could pass } \\
\text { coronavirus to someone else }\end{array}$ & $\begin{array}{l}\text { 5-point scale, } \\
1=\text { strongly disagree } \\
\text { to } 5=\text { strongly agree }\end{array}$ & $\begin{array}{l}\mathrm{N}=1607 \\
\mathrm{M}=4 \cdot 39 \\
\mathrm{SD}=0 \cdot 85\end{array}$ & $\begin{array}{l}\mathrm{N}=455 \\
\mathrm{M}=4 \cdot 38 \\
\mathrm{SD}=0 \cdot 87\end{array}$ & $\begin{array}{l}0.98(0 \cdot 87 \text { to } \\
1 \cdot 11)\end{array}$ & $\begin{array}{l}0.96(0.83 \text { to } \\
1 \cdot 10)\end{array}$ & $\begin{array}{l}\mathrm{N}=1586 \\
\mathrm{M}=4 \cdot 40 \\
\mathrm{SD}=0 \cdot 81\end{array}$ & $\begin{array}{l}\mathrm{N}=492 \\
\mathrm{M}=4 \cdot 31 \\
\mathrm{SD}=0.94\end{array}$ & $\begin{array}{l}0.88(0.79 \text { to } \\
0.99)^{*}\end{array}$ & $\begin{array}{l}0.89(0.78 \text { to } \\
1.01)\end{array}$ \\
\hline $\begin{array}{l}\text { If I leave home and meet } \\
\text { other people, I could catch } \\
\text { coronavirus }\end{array}$ & $\begin{array}{l}\text { 5-point scale, } \\
1=\text { strongly disagree } \\
\text { to } 5=\text { strongly agree }\end{array}$ & $\begin{array}{l}\mathrm{N}=1618 \\
\mathrm{M}=4 \cdot 39 \\
\mathrm{SD}=0 \cdot 77\end{array}$ & $\begin{array}{l}\mathrm{N}=456 \\
\mathrm{M}=4 \cdot 36 \\
\mathrm{SD}=0 \cdot 85\end{array}$ & $\begin{array}{l}0.95(0.84 \text { to } \\
1.08)\end{array}$ & $\begin{array}{l}0.95(0.82 \text { to } \\
1 \cdot 11)\end{array}$ & $\begin{array}{l}\mathrm{N}=1596 \\
\mathrm{M}=4 \cdot 41 \\
\mathrm{SD}=0 \cdot 74\end{array}$ & $\begin{array}{l}\mathrm{N}=492 \\
\mathrm{M}=4 \cdot 26 \\
\mathrm{SD}=0 \cdot 93\end{array}$ & $\begin{array}{l}0.79(0.70 \text { to } \\
0 \cdot 89)^{* *}\end{array}$ & $\begin{array}{l}0.81(0.70 \text { to } \\
0.93)^{*}\end{array}$ \\
\hline $\begin{array}{l}\text { If I follow the Government's } \\
\text { advice it will have a negative } \\
\text { impact on how much money I } \\
\text { have }\end{array}$ & $\begin{array}{l}\text { 5-point scale, } \\
1=\text { strongly disagree } \\
\text { to } 5=\text { strongly agree }\end{array}$ & $\begin{array}{l}\mathrm{N}=1603 \\
\mathrm{M}=2 \cdot 44 \\
\mathrm{SD}=1 \cdot 20\end{array}$ & $\begin{array}{l}\mathrm{N}=444 \\
\mathrm{M}=2 \cdot 97 \\
\mathrm{SD}=1 \cdot 25\end{array}$ & $\begin{array}{l}1.41(1.29 \text { to } \\
1.53)^{* *}\end{array}$ & $\begin{array}{l}1.41(1.28 \text { to } \\
1.55)^{* *}\end{array}$ & $\begin{array}{l}\mathrm{N}=1580 \\
\mathrm{M}=2 \cdot 44 \\
\mathrm{SD}=1 \cdot 19\end{array}$ & $\begin{array}{l}\mathrm{N}=480 \\
\mathrm{M}=2 \cdot 99 \\
\mathrm{SD}=1 \cdot 26\end{array}$ & $\begin{array}{l}1.44(1.32 \text { to } \\
1.56)^{* * *}\end{array}$ & $\begin{array}{l}1.42(1.29 \text { to } \\
1.56)^{* *}\end{array}$ \\
\hline $\begin{array}{l}\text { Because of the current } \\
\text { lockdown there is more } \\
\text { conflict between people that I } \\
\text { live with }\end{array}$ & $\begin{array}{l}\text { 5-point scale, } \\
1=\text { strongly disagree } \\
\text { to } 5=\text { strongly agree }\end{array}$ & $\begin{array}{l}\mathrm{N}=1573 \\
\mathrm{M}=2 \cdot 04 \\
\mathrm{SD}=1 \cdot 10\end{array}$ & $\begin{array}{l}\mathrm{N}=444 \\
\mathrm{M}=2 \cdot 72 \\
\mathrm{SD}=1 \cdot 30\end{array}$ & $\begin{array}{l}1.59(1.46 \text { to } \\
1.74)^{* *}\end{array}$ & $\begin{array}{l}1.54(1.39 \text { to } \\
1.71)^{* *}\end{array}$ & $\begin{array}{l}\mathrm{N}=1555 \\
\mathrm{M}=2 \cdot 03 \\
\mathrm{SD}=1 \cdot 10\end{array}$ & $\begin{array}{l}\mathrm{N}=477 \\
\mathrm{M}=2 \cdot 81 \\
\mathrm{SD}=1 \cdot 28\end{array}$ & $\begin{array}{l}1.71(1.57 \text { to } \\
1.87)^{* * *}\end{array}$ & $\begin{array}{l}1.59(1.44 \text { to } \\
1.76)^{* *}\end{array}$ \\
\hline If I follow the Government's & 5-point scale, & $\mathrm{N}=1451$ & $\mathrm{~N}=406$, & $1.01(0.93$ to & $1.05(0.95$ to & $\mathrm{N}=1432$ & $\mathrm{~N}=431$, & $1.07(0.98$ to & $1.10(1.00$ to \\
\hline
\end{tabular}




\begin{tabular}{|c|c|c|c|c|c|c|c|c|c|}
\hline $\begin{array}{l}\text { advice I will not be able to } \\
\text { carry out important religious } \\
\text { activities }\end{array}$ & $\begin{array}{l}1=\text { strongly disagree } \\
\text { to } 5=\text { strongly agree }\end{array}$ & $\begin{array}{l}\mathrm{M}=2 \cdot 61 \\
\mathrm{SD}=1 \cdot 35\end{array}$ & $\begin{array}{l}\mathrm{M}=2 \cdot 63 \\
\mathrm{SD}=1 \cdot 32\end{array}$ & 1.10) & $1 \cdot 15)$ & $\begin{array}{l}\mathrm{M}=2 \cdot 59 \\
\mathrm{SD}=1 \cdot 34\end{array}$ & $\begin{array}{l}\mathrm{M}=2 \cdot 70 \\
\mathrm{SD}=1 \cdot 34\end{array}$ & 1.15) & $1.20)^{*}$ \\
\hline $\begin{array}{l}\text { I am enjoying spending more } \\
\text { time at home during the } \\
\text { lockdown }\end{array}$ & $\begin{array}{l}\text { 5-point scale, } \\
1=\text { strongly disagree } \\
\text { to } 5=\text { strongly agree }\end{array}$ & $\begin{array}{l}\mathrm{N}=1628 \\
\mathrm{M}=3 \cdot 4 \\
\mathrm{SD}=1 \cdot 17\end{array}$ & $\begin{array}{l}\mathrm{N}=454 \\
\mathrm{M}=2 \cdot 92 \\
\mathrm{SD}=1 \cdot 30\end{array}$ & $\begin{array}{l}0.72(0.66 \text { to } \\
0.79)^{* *}\end{array}$ & $\begin{array}{l}0.69(0.62 \text { to } \\
0.76)^{* *}\end{array}$ & $\begin{array}{l}\mathrm{N}=1605, \\
\mathrm{M}=3 \cdot 42, \\
\mathrm{SD}=1 \cdot 15\end{array}$ & $\begin{array}{l}\mathrm{N}=490 \\
\mathrm{M}=2 \cdot 77 \\
\mathrm{SD}=1 \cdot 29\end{array}$ & $\begin{array}{l}0.64(0.59 \text { to } \\
0.70)^{* *}\end{array}$ & $\begin{array}{l}0.61(0.55 \text { to } \\
0.68)^{* *}\end{array}$ \\
\hline $\begin{array}{l}\text { Because of coronavirus, I feel } \\
\text { a sense of community with } \\
\text { other people in my } \\
\text { neighbourhood }\end{array}$ & $\begin{array}{l}\text { 5-point scale, } \\
1=\text { strongly disagree } \\
\text { to } 5=\text { strongly agree }\end{array}$ & $\begin{array}{l}\mathrm{N}=1620 \\
\mathrm{M}=3 \cdot 35 \\
\mathrm{SD}=1 \cdot 05\end{array}$ & $\begin{array}{l}\mathrm{N}=453 \\
\mathrm{M}=3 \cdot 08 \\
\mathrm{SD}=1 \cdot 26\end{array}$ & $\begin{array}{l}0.81(0.74 \text { to } \\
0.89)^{* *}\end{array}$ & $\begin{array}{l}0.83(0.74 \text { to } \\
0.92)^{* *}\end{array}$ & $\begin{array}{l}\mathrm{N}=1599, \\
\mathrm{M}=3 \cdot 38 \\
\mathrm{SD}=1 \cdot 03\end{array}$ & $\begin{array}{l}\mathrm{N}=489 \\
\mathrm{M}=2 \cdot 99 \\
\mathrm{SD}=1 \cdot 27\end{array}$ & $\begin{array}{l}0.73(0.67 \text { to } \\
0.80)^{* *}\end{array}$ & $\begin{array}{l}0.77(0.69 \text { to } \\
0.85)^{* *}\end{array}$ \\
\hline
\end{tabular}

$* p \leq .05$
$* * p<.001$

$* * p \leq .001$

$\dagger$ Adjusting for gender, age, having a child in the household, being extremely clinically vulnerable oneself, employment status, highest level of education or professional qualification, indices of multiple deprivation, social grade, living in a rural or urban area, living alone, marital status, and region. 
medRxiv preprint doi: https://doi.org/10.1101/2020.06.23.20137901; this version posted June 23, 2020. The copyright holder for this preprint (which was not certified by peer review) is the author/funder, who has granted medRxiv a license to display the preprint in perpetuity.

It is made available under a CC-BY 4.0 International license.

\section{Depression}

Data were available to calculate depression scores for 2103 participants. $23.5 \%(n=494,95 \%$ CI [21.7\% to 25.3]) reported probable depression (scored three or over on PHQ-2).

Depression was associated with younger age, being clinically extremely vulnerable to COVID-19, not working, living in a more deprived area (Table 1). Those with a household member who was clinically extremely vulnerable to COVID-19 were less likely to report depression.

Depression was also associated with: presence of anxiety; feeling that the current lockdown had made your mental and physical health worse; presence of COVID-19 symptoms in the household; not enjoying spending more time at home because of the lockdown; more conflict in your household as a result of the current lockdown; believing that following Government advice would impact your finances negatively; cause you to lose touch with friends and relatives, and stop you from being able to carry out important religious activities; lower perceived effectiveness of Government measures; lower perceived sense of community with others in your neighbourhood; greater perceived likelihood of catching COVID-19; greater worry about COVID-19; greater perceived disapproval from family and friends if you did not follow Government advice; and thinking that fewer people of your age in the UK were following Government recommendations to stay at home (lower perceived social norms; Table 2).

\section{Self-reported general health}

Self-reported general health was approximately normally distributed $(\mathrm{N}=2208, \mathrm{M}=2 \cdot 99$, $\mathrm{SD}=1 \cdot 04$, median $=3$, mode $=3$ ).

Personal and clinical factors (gender, age, having a child in the household, being extremely clinically vulnerable oneself, employment status, highest level of education or professional qualification, indices of multiple deprivation, social grade, living in a rural or urban area, living alone, marital status, and region [results for region not reported]) explained $9.3 \%$ of the variance in self-reported general health (Table 3). Poorer self-reported general health was associated with being extremely clinically vulnerable to COVID-19 oneself, living in a more deprived area and lower social grade. Better self-reported general health was associated with being employed and being more educated (having a degree or higher). 
Poorer self-reported general health was also associated with: thinking that the current lockdown had made your physical and mental health worse; presence of depression; presence of anxiety; reporting that you were currently self-isolating; greater perceived severity of COVID-19 (to self and family's wellbeing); more conflict in your household as a result of the current lockdown; greater worry about COVID-19; presence of COVID-19 symptoms in the household; having received help from someone outside your household because of COVID19; greater perceived likelihood of catching COVID-19; lower perceived effectiveness of Government measures; believing that following Government advice would impact your finances negatively; and thinking that you have had or currently have COVID-19 (Table 4). Factors associated with better self-reported general health were: greater total out of home activity; feeling an greater sense of community with people in your neighbourhood; thinking that more people of your age in the UK were following Government recommendations to stay at home (greater perceived social norms); enjoying spending more time at home during the lockdown; and having helped someone outside your household because of COVID-19 in the last week. 
Table 3. Associations between personal and clinical characteristics and self-reported general health.

\begin{tabular}{|c|c|c|c|c|c|c|c|c|c|c|c|c|}
\hline \multirow[t]{2}{*}{$\begin{array}{l}\text { Participant } \\
\text { characteristics }\end{array}$} & \multirow[t]{2}{*}{ Level } & \multirow[t]{2}{*}{$\begin{array}{l}\text { Self-reported } \\
\text { general health }\end{array}$} & \multicolumn{3}{|c|}{$\begin{array}{l}\text { Self-reported general health } \\
\text { Unadjusted analyses } \\
\text { Model }\end{array}$} & \multicolumn{2}{|c|}{$\begin{array}{l}\text { Regression } \\
\text { coefficient }\end{array}$} & \multicolumn{2}{|c|}{$\begin{array}{l}\text { Adjusted analyses } \dagger \\
\text { Model }\end{array}$} & \multirow[b]{2}{*}{$p$} & \multicolumn{2}{|c|}{$\begin{array}{l}\text { Regression } \\
\text { coefficient }\end{array}$} \\
\hline & & & $F$ & $\begin{array}{l}\text { Adjuste } \\
\mathrm{d} \mathrm{R}^{2}\end{array}$ & $p$ & $\beta$ & $p$ & $F$ & $\begin{array}{l}\text { Adjusted } \\
\mathrm{R}^{2}\end{array}$ & & $\beta$ & $p$ \\
\hline \multirow[t]{2}{*}{ Gender } & Male, $\mathrm{n}=1074$ & $\mathrm{M}=2.99, \mathrm{SD}=1.03$ & & & & & & & & & & \\
\hline & Female, $n=1134$ & $\mathrm{M}=2.99, \mathrm{SD}=1.05$ & $0 \cdot 00$ & .000 & .96 & .00 & .96 & & & & -.01 & .64 \\
\hline \multirow[t]{5}{*}{ Age } & 18 to 24 years, $n=240$ & $\mathrm{M}=3 \cdot 07, \mathrm{SD}=0 \cdot 85$ & & & & & & & & & & \\
\hline & 25 to 34 years, $n=320$ & $\mathrm{M}=3.09, \mathrm{SD}=1.00$ & & & & & & & & & & \\
\hline & 35 to 44 years, $n=392$ & $\mathrm{M}=2.98, \mathrm{SD}=1.05$ & & & & & & & & & & \\
\hline & 45 to 54 years, $n=370$ & $\mathrm{M}=2.93, \mathrm{SD}=1.09$ & & & & & & & & & & \\
\hline & 55 years and over, $\mathrm{n}=886$ & $\mathrm{M}=2.97, \mathrm{SD}=1.07$ & $4 \cdot 01$ & .001 & $.05^{*}$ & -.04 & $.05^{*}$ & & & & -.04 & .14 \\
\hline \multirow[t]{2}{*}{ Child in the household } & None, $\mathrm{n}=1574$ & $\mathrm{M}=2.98, \mathrm{SD}=1.05$ & & & & & & & & & & \\
\hline & Child present, $\mathrm{n}=597$ & $\mathrm{M}=3.07, \mathrm{SD}=1.01$ & $3 \cdot 61$ & .001 & .06 & .04 & .06 & & & & -.02 & .48 \\
\hline \multirow{2}{*}{$\begin{array}{l}\text { Clinically extremely } \\
\text { vulnerable (self) }\end{array}$} & No, $n=1989$ & $\mathrm{M}=3.05, \mathrm{SD}=1.01$ & & & & & & & & & & \\
\hline & Yes, $n=147$ & $\mathrm{M}=2 \cdot 31, \mathrm{SD}=1 \cdot 20$ & $72 \cdot 20$ & .032 & $<.001 * *$ & $-\cdot 18$ & $<.001 * *$ & & & & $-\cdot 15$ & $<\cdot 001 * *$ \\
\hline \multirow[t]{2}{*}{ Employment status } & Not working, $\mathrm{n}=1025$ & $\mathrm{M}=2 \cdot 85, \mathrm{SD}=1 \cdot 07$ & & & & & & & & & & \\
\hline & Working, $\mathrm{n}=1183$ & $\mathrm{M}=3 \cdot 12, \mathrm{SD}=0 \cdot 99$ & $37 \cdot 01$ & .016 & $<\cdot 001 * *$ & $\cdot 13$ & $<\cdot 001 * *$ & & & & $\cdot 10$ & $<\cdot 001 * *$ \\
\hline \multirow{2}{*}{$\begin{array}{l}\text { Highest educational or } \\
\text { professional } \\
\text { qualification }\end{array}$} & $\begin{array}{l}\text { GCSE/vocational/A-level/No } \\
\text { formal qualifications, } n=1040\end{array}$ & $\mathrm{M}=2 \cdot 88, \mathrm{SD}=0 \cdot 98$ & & & & & & & & & & \\
\hline & $\begin{array}{l}\text { Degree or higher (Bachelors, } \\
\text { Masters, } \mathrm{PhD}), \mathrm{n}=1125\end{array}$ & $\mathrm{M}=3 \cdot 11, \mathrm{SD}=1 \cdot 07$ & $25 \cdot 08$ & $\cdot 011$ & $<.001 * *$ & $\cdot 11$ & $<.001 * *$ & & & & .07 & $.003 *$ \\
\hline IMD decile & $\begin{array}{l}\text { Range } 1 \text { (most deprived) to } 10 \\
\text { (least deprived), } n=2208\end{array}$ & $\mathrm{M}=2 \cdot 99, \mathrm{SD}=1 \cdot 04$ & $63 \cdot 42$ & .028 & $<\cdot 001 * *$ & $\cdot 17$ & $<\cdot 001 * *$ & & & & $\cdot 15$ & $<\cdot 001 *$ \\
\hline \multirow[t]{2}{*}{ Social grade } & $\mathrm{ABC} 1, \mathrm{n}=1266$ & $\mathrm{M}=3 \cdot 15, \mathrm{SD}=0 \cdot 97$ & & & & & & & & & & \\
\hline & C2DE, n=942 & $\mathrm{M}=2 \cdot 77, \mathrm{SD}=1 \cdot 08$ & $75 \cdot 22$ & .033 & $<\cdot 001 * *$ & $-\cdot 18$ & $<\cdot 001 * *$ & & & & $-\cdot 11$ & $<\cdot 001 * *$ \\
\hline \multirow[t]{2}{*}{ Urban/rural } & Urban, $n=1693$ & $\mathrm{M}=2.98, \mathrm{SD}=1.03$ & & & & & & & & & & \\
\hline & Rural, $n=456$ & $\mathrm{M}=3 \cdot 06, \mathrm{SD}=1 \cdot 04$ & $2 \cdot 22$ & .001 & .14 & .03 & $\cdot 14$ & & & & .01 & .59 \\
\hline \multirow[t]{2}{*}{ Living alone } & Yes, $n=421$ & $\mathrm{M}=2 \cdot 81, \mathrm{SD}=1 \cdot 09$ & & & & & & & & & & \\
\hline & No, $n=1784$ & $\mathrm{M}=3 \cdot 04, \mathrm{SD}=1 \cdot 02$ & $16 \cdot 85$ & .007 & $<.001 * *$ & .09 & $<\cdot 001 * *$ & & & & .03 & .24 \\
\hline \multirow[t]{2}{*}{ Marital status } & $\begin{array}{l}\text { Married/civil partnership/living } \\
\text { as married, } n=1292\end{array}$ & $\mathrm{M}=3 \cdot 07, \mathrm{SD}=1 \cdot 03$ & & & & & & & & & & \\
\hline & $\begin{array}{l}\text { Separated/divorced/widowed/ne } \\
\text { ver married, } n=898\end{array}$ & $\mathrm{M}=2 \cdot 89, \mathrm{SD}=1 \cdot 04$ & $15 \cdot 43$ & .007 & $<\cdot 001 * *$ & -.08 & $<.001 * *$ & & & & -.04 & .13 \\
\hline Model & & & - & - & - & - & - & 17.98 & .093 & $<.001 * *$ & & \\
\hline $\begin{array}{l}\text { Clinically extremely } \\
\text { vulnerable (household } \\
\text { member) } \ddagger\end{array}$ & $\begin{array}{l}\text { No, } n=1563 \\
\text { Yes, } n=164\end{array}$ & $\begin{array}{l}\mathrm{M}=3 \cdot 04, \mathrm{SD}=1 \cdot 03 \\
\mathrm{M}=3 \cdot 10, \mathrm{SD}=0 \cdot 94\end{array}$ & 0.56 & .000 & .46 & .02 & .46 & $13 \cdot 35$ & .084 & $<\cdot 001 * *$ & .01 & .64 \\
\hline
\end{tabular}




\begin{tabular}{|c|c|c|c|c|c|c|c|c|c|c|c|c|}
\hline $\begin{array}{l}\text { Home includes access } \\
\text { to outside space }\end{array}$ & $\begin{array}{l}\text { No, } n=174 \\
\text { Yes, } n=2034\end{array}$ & $\begin{array}{l}\mathrm{M}=2 \cdot 77, \mathrm{SD}=1 \cdot 11 \\
\mathrm{M}=3 \cdot 01, \mathrm{SD}=1 \cdot 03\end{array}$ & 8.77 & .004 & $.003^{*}$ & .06 & $.003^{*}$ & 16.68 & .093 & $<\cdot 001^{* * *}$ & .02 & .29 \\
\hline Pet ownership & $\begin{array}{l}\text { No, } n=1180 \\
\text { Yes, } n=1028\end{array}$ & $\begin{array}{l}\mathrm{M}=2 \cdot 99, \mathrm{SD}=1 \cdot 03 \\
\mathrm{M}=3 \cdot 00, \mathrm{SD}=1 \cdot 04\end{array}$ & 0.04 & .000 & .84 & .00 & .84 & 16.59 & .092 & $<\cdot 001 * *$ & .00 & .90 \\
\hline
\end{tabular}

$* p \leq-05$

$* * p \leq-001$

$\dagger$ Adjusting for gender, age, having a child in the household, being extremely clinically vulnerable oneself, employment status, highest level of education or professional qualification, indices of multiple deprivation, social grade, living in a rural or urban area, living alone, marital status, and region. Personal and clinical characteristics entered as first block, other independent variables entered as second block.

¥Adjusted analyses for this variable did not control for living alone, as by definition all participants asked this question lived in a household with someone else. 
Table 4. Associations between psychological and situational factors and self-reported general health.

\begin{tabular}{|c|c|c|c|c|c|c|c|c|c|c|c|c|}
\hline \multirow[t]{2}{*}{ Participant characteristics } & \multirow[t]{2}{*}{ Level } & \multirow[t]{2}{*}{$\begin{array}{l}\text { Self-reported } \\
\text { general health }\end{array}$} & \multicolumn{3}{|c|}{$\begin{array}{l}\text { Self-reported general health } \\
\text { Unadjusted analyses } \\
\text { Model }\end{array}$} & \multicolumn{2}{|c|}{$\begin{array}{l}\text { Regression } \\
\text { coefficient }\end{array}$} & \multicolumn{2}{|c|}{$\begin{array}{l}\text { Adjusted analyses } \dagger \\
\text { Model }\end{array}$} & \multirow[b]{2}{*}{$p$} & \multicolumn{2}{|c|}{$\begin{array}{l}\text { Regression } \\
\text { coefficient }\end{array}$} \\
\hline & & & $F$ & $\begin{array}{l}\text { Adjusted } \\
\mathrm{R}^{2}\end{array}$ & $p$ & $\beta$ & $p$ & $F$ & $\begin{array}{l}\text { Adjusted } \\
\mathrm{R}^{2}\end{array}$ & & $\beta$ & $p$ \\
\hline \multirow[t]{2}{*}{ Probable anxiety } & None, $n=1625$ & $\mathrm{M}=3 \cdot 10, \mathrm{SD}=1 \cdot 01$ & & & & & & & & & & \\
\hline & Present, $n=446$ & $\mathrm{M}=2 \cdot 62, \mathrm{SD}=1.06$ & $78 \cdot 32$ & .036 & $<.001 * *$ & $-\cdot 19$ & $<.001 * *$ & $19 \cdot 48$ & $\cdot 112$ & $<.001 * *$ & $-\cdot 16$ & $<.001 * *$ \\
\hline \multirow[t]{2}{*}{ Probable depression } & None, $n=1604$ & $\mathrm{M}=3 \cdot 12, \mathrm{SD}=1 \cdot 01$ & & & & & & & & & & \\
\hline & Present, $n=485$ & $\mathrm{M}=2 \cdot 58, \mathrm{SD}=1.05$ & 103.99 & .047 & $<.001 * *$ & -.22 & $<.001 * *$ & 22.72 & .129 & $<.001 * *$ & -.19 & $<\cdot 001 * *$ \\
\hline \multirow[t]{2}{*}{$\begin{array}{l}\text { Had, or currently have, } \\
\text { COVID-19 }\end{array}$} & $\begin{array}{l}\text { Think have not had } \\
\text { COVID-19 and do not } \\
\text { have it now, } n=1680\end{array}$ & $\mathrm{M}=3 \cdot 03, \mathrm{SD}=1 \cdot 04$ & & & & & & & & & & \\
\hline & $\begin{array}{l}\text { Think have had COVID- } \\
19 \text { or have it now, } \\
\mathrm{n}=224\end{array}$ & $\mathrm{M}=2 \cdot 88, \mathrm{SD}=1 \cdot 02$ & $3 \cdot 93$ & .002 & $.05^{*}$ & -.05 & $.05^{*}$ & 13.92 & .088 & $<\cdot 001 * *$ & -.05 & $.02 *$ \\
\hline \multirow[t]{2}{*}{$\begin{array}{l}\text { Symptoms of COVID-19 in } \\
\text { the household }\end{array}$} & $\begin{array}{l}\text { No symptoms present, } \\
\mathrm{n}=1906\end{array}$ & $\mathrm{M}=3 \cdot 04, \mathrm{SD}=1 \cdot 03$ & & & & & & & & & & \\
\hline & $\begin{array}{l}\text { Symptoms present, } \\
\mathrm{n}=218\end{array}$ & $\mathrm{M}=2 \cdot 69, \mathrm{SD}=1 \cdot 00$ & $22 \cdot 67$ & .010 & $<\cdot 001 * *$ & $-\cdot 10$ & $<.001 * *$ & $18 \cdot 01$ & $\cdot 109$ & $<.001 * *$ & $-\cdot 10$ & $<\cdot 001 * *$ \\
\hline \multirow[t]{2}{*}{ Self-isolating } & $\begin{array}{l}\text { Not self-isolating, } \\
\mathrm{n}=1679\end{array}$ & $\mathrm{M}=3 \cdot 12, \mathrm{SD}=0 \cdot 99$ & & & & & & & & & & \\
\hline & Self-isolating, $n=529$ & $\mathrm{M}=2 \cdot 60, \mathrm{SD}=1.08$ & $106 \cdot 39$ & .046 & $<\cdot 001 * *$ & -.22 & $<.001 * *$ & $20 \cdot 04$ & $\cdot 110$ & $<\cdot 001 * *$ & $-\cdot 14$ & $<.001 * *$ \\
\hline $\begin{array}{l}\text { Total out of home activity } \\
\text { in past week }\end{array}$ & Range 0 to $20+$ & $\mathrm{M}=2 \cdot 99, \mathrm{SD}=1.04$ & 74.90 & .032 & $<\cdot 001^{* *}$ & $\cdot 18$ & $<\cdot 001 * *$ & $20 \cdot 02$ & $\cdot 110$ & $<\cdot 001 * *$ & $\cdot 14$ & $<\cdot 001 * *$ \\
\hline Worry about COVID-19 & $\begin{array}{l}5 \text {-point scale, } 1=\text { not at } \\
\text { all worried to } \\
5=\text { extremely worried, } \\
n=2199\end{array}$ & $\mathrm{M}=2 \cdot 99, \mathrm{SD}=1 \cdot 04$ & $39 \cdot 41$ & .017 & $<\cdot 001 * *$ & $-\cdot 13$ & $<.001 * *$ & 18.98 & $\cdot 105$ & $<\cdot 001 * *$ & $-\cdot 11$ & $<\cdot 001 * *$ \\
\hline Perceived social norms & $\begin{array}{l}\text { Percentage (range } 0- \\
100), n=1977\end{array}$ & $\mathrm{M}=3 \cdot 03, \mathrm{SD}=1 \cdot 03$ & $22 \cdot 62$ & .011 & $<\cdot 001 * *$ & $\cdot 11$ & $<\cdot 001 * *$ & 14.99 & .091 & $<\cdot 001 * *$ & .08 & $<\cdot 001 * *$ \\
\hline $\begin{array}{l}\text { Perceptions about impact on } \\
\text { mental health }\end{array}$ & $\begin{array}{l}5 \text {-point scale, } 1=a \text { lot } \\
\text { better to } 5=a \text { lot worse, } \\
n=2180\end{array}$ & $\mathrm{M}=3 \cdot 00, \mathrm{SD}=1 \cdot 03$ & $58 \cdot 75$ & .026 & $<\cdot 001 * *$ & $-\cdot 16$ & $<\cdot 001 * *$ & $20 \cdot 56$ & $\cdot 114$ & $<\cdot 001 * *$ & $-\cdot 16$ & $<\cdot 001 * *$ \\
\hline $\begin{array}{l}\text { Perceptions about impact on } \\
\text { physical health }\end{array}$ & $\begin{array}{l}5 \text {-point scale, } 1=a \text { lot } \\
\text { better to } 5=a \text { lot worse, } \\
n=2189\end{array}$ & $\mathrm{M}=3 \cdot 00, \mathrm{SD}=1 \cdot 03$ & $127 \cdot 77$ & .055 & $<\cdot 001 * *$ & $-\cdot 24$ & $<\cdot 001 * *$ & $25 \cdot 13$ & $\cdot 136$ & $<\cdot 001 * *$ & -.22 & $<\cdot 001 * *$ \\
\hline \multirow{2}{*}{$\begin{array}{l}\text { Helped someone outside } \\
\text { household }\end{array}$} & No, $n=1682$ & $\mathrm{M}=2 \cdot 94, \mathrm{SD}=1 \cdot 03$ & & & & & & & & & & \\
\hline & Yes, $\mathrm{n}=508$ & $\mathrm{M}=3 \cdot 17, \mathrm{SD}=1 \cdot 03$ & $18 \cdot 81$ & .008 & $<.001 * *$ & .09 & $<.001 * *$ & $17 \cdot 24$ & .096 & $<\cdot 001 * *$ & .06 & $.004 *$ \\
\hline
\end{tabular}




\begin{tabular}{|c|c|c|c|c|c|c|c|c|c|c|c|c|}
\hline $\begin{array}{l}\text { Received help from } \\
\text { someone outside household }\end{array}$ & $\begin{array}{l}\text { No, } n=1903 \\
\text { Yes, } n=288\end{array}$ & $\begin{array}{l}\mathrm{M}=3 \cdot 04, \mathrm{SD}=1 \cdot 02 \\
\mathrm{M}=2 \cdot 68, \mathrm{SD}=1 \cdot 06\end{array}$ & $31 \cdot 39$ & .014 & $<.001 * *$ & $-\cdot 12$ & $<.001 * *$ & $17 \cdot 76$ & .099 & $<.001 * *$ & -.09 & $<.001 * *$ \\
\hline $\begin{array}{l}\text { If I completely follow the } \\
\text { Government's advice, I will } \\
\text { lose touch with my friends } \\
\text { and relatives }\end{array}$ & $\begin{array}{l}\text { 5-point scale, } 1=\text { strongly } \\
\text { disagree to } 5=\text { strongly } \\
\text { agree, } n=2188\end{array}$ & $\mathrm{M}=3 \cdot 00, \mathrm{SD}=1 \cdot 03$ & 3.98 & .001 & $.05^{*}$ & -.04 & $.05^{*}$ & $16 \cdot 46$ & .092 & $<.001 * *$ & -.04 & .07 \\
\hline $\begin{array}{l}\text { My friends or family will } \\
\text { disapprove if I don't follow } \\
\text { the Government's advice }\end{array}$ & $\begin{array}{l}5 \text {-point scale, } 1=\text { strongly } \\
\text { disagree to } 5=\text { strongly } \\
\text { agree, } n=2156\end{array}$ & $\mathrm{M}=3 \cdot 00, \mathrm{SD}=1 \cdot 03$ & $1 \cdot 88$ & .000 & $\cdot 17$ & .03 & .17 & $15 \cdot 57$ & .088 & $<.001 * *$ & .02 & .50 \\
\hline $\begin{array}{l}\text { If I don't follow the } \\
\text { Government's advice, I } \\
\text { could get in trouble with the } \\
\text { police }\end{array}$ & $\begin{array}{l}5 \text {-point scale, } 1=\text { strongly } \\
\text { disagree to } 5=\text { strongly } \\
\text { agree, } n=2172\end{array}$ & $\mathrm{M}=3 \cdot 00, \mathrm{SD}=1 \cdot 03$ & $0 \cdot 73$ & .000 & .39 & -.02 & .39 & $16 \cdot 78$ & .094 & $<\cdot 001 * *$ & .00 & .99 \\
\hline $\begin{array}{l}\text { If I follow the } \\
\text { Government's advice, it } \\
\text { will help save lives }\end{array}$ & $\begin{array}{l}5 \text {-point scale, } 1=\text { strongly } \\
\text { disagree to } 5=\text { strongly } \\
\text { agree, } n=2189\end{array}$ & $\mathrm{M}=3 \cdot 00, \mathrm{SD}=1 \cdot 03$ & $2 \cdot 14$ & .001 & .14 & -.03 & $\cdot 14$ & $16 \cdot 77$ & .094 & $<.001 * *$ & -.05 & $.03 *$ \\
\hline $\begin{array}{l}\text { If I follow the } \\
\text { Government's advice, it } \\
\text { will help protect the NHS }\end{array}$ & $\begin{array}{l}5 \text {-point scale, } 1=\text { strongly } \\
\text { disagree to } 5=\text { strongly } \\
\text { agree, } n=2192\end{array}$ & $\mathrm{M}=2.99, \mathrm{SD}=1.04$ & $0 \cdot 12$ & .000 & .90 & .00 & .90 & $16 \cdot 59$ & .093 & $<\cdot 001 * *$ & -.02 & .32 \\
\hline $\begin{array}{l}\text { If I catch coronavirus, I may } \\
\text { become very ill }\end{array}$ & $\begin{array}{l}5 \text {-point scale, } 1=\text { strongly } \\
\text { disagree to } 5=\text { strongly } \\
\text { agree, } n=2166\end{array}$ & $\mathrm{M}=2.99, \mathrm{SD}=1.03$ & $37 \cdot 96$ & .017 & $<-001 * *$ & $-\cdot 13$ & $<\cdot 001 * *$ & $18 \cdot 46$ & $\cdot 104$ & $<.001 * *$ & $-\cdot 11$ & $<\cdot 001 * *$ \\
\hline $\begin{array}{l}\text { If I catch coronavirus, it } \\
\text { will have a severe impact } \\
\text { on my family's wellbeing }\end{array}$ & $\begin{array}{l}\text { 5-point scale, } 1=\text { strongly } \\
\text { disagree to } 5=\text { strongly } \\
\text { agree, } n=2151\end{array}$ & $\mathrm{M}=3.00, \mathrm{SD}=1 \cdot 03$ & $7 \cdot 50$ & .003 & $.01 *$ & -.06 & $.01 *$ & $16 \cdot 87$ & .096 & $<\cdot 001 * *$ & -.05 & $.02 *$ \\
\hline $\begin{array}{l}\text { If I leave home and meet } \\
\text { other people, I could pass } \\
\text { coronavirus to someone else }\end{array}$ & $\begin{array}{l}5 \text {-point scale, } 1=\text { strongly } \\
\text { disagree to } 5=\text { strongly } \\
\text { agree, } n=2180\end{array}$ & $\mathrm{M}=2.99, \mathrm{SD}=1.04$ & 0.92 & .000 & .34 & -.02 & .34 & $16 \cdot 33$ & .092 & $<.001 * *$ & -.02 & .33 \\
\hline $\begin{array}{l}\text { If I leave home and meet } \\
\text { other people, I could catch } \\
\text { coronavirus }\end{array}$ & $\begin{array}{l}5 \text {-point scale, } 1=\text { strongly } \\
\text { disagree to } 5=\text { strongly } \\
\text { agree, } n=2190\end{array}$ & $\mathrm{M}=2.99, \mathrm{SD}=1.03$ & 14.94 & .006 & $<\cdot 001 * *$ & -.08 & $<\cdot 001 * *$ & $17 \cdot 05$ & .095 & $<\cdot 001 * *$ & -.06 & $.004 *$ \\
\hline $\begin{array}{l}\text { If I follow the } \\
\text { Government's advice it will } \\
\text { have a negative impact on } \\
\text { how much money I have }\end{array}$ & $\begin{array}{l}5 \text {-point scale, } 1=\text { strongly } \\
\text { disagree to } 5=\text { strongly } \\
\text { agree, } n=2152\end{array}$ & $\mathrm{M}=3 \cdot 00, \mathrm{SD}=1 \cdot 04$ & 13.94 & .006 & $<\cdot 001 * *$ & -.08 & $<\cdot 001 * *$ & $16 \cdot 00$ & .091 & $<\cdot 001 * *$ & -.05 & $.02 *$ \\
\hline $\begin{array}{l}\text { Because of the current } \\
\text { lockdown there is more } \\
\text { conflict between people that } \\
\text { I live with }\end{array}$ & $\begin{array}{l}5 \text {-point scale, } 1=\text { strongly } \\
\text { disagree to } 5=\text { strongly } \\
\text { agree, } n=2125\end{array}$ & $\mathrm{M}=3.00, \mathrm{SD}=1 \cdot 03$ & $38 \cdot 36$ & .017 & $<\cdot 001 * *$ & $-\cdot 13$ & $<\cdot 001 * *$ & 18.97 & $\cdot 108$ & $<.001 * *$ & $-\cdot 13$ & $<\cdot 001 * *$ \\
\hline $\begin{array}{l}\text { If I follow the } \\
\text { Government's advice I will } \\
\text { not be able to carry out }\end{array}$ & $\begin{array}{l}5 \text {-point scale, } 1=\text { strongly } \\
\text { disagree to } 5=\text { strongly } \\
\text { agree, } n=1957\end{array}$ & $\mathrm{M}=3 \cdot 00, \mathrm{SD}=1 \cdot 04$ & $0 \cdot 48$ & .000 & .49 & -.02 & .49 & $14 \cdot 56$ & .093 & $<.001 * *$ & .02 & .45 \\
\hline
\end{tabular}




\begin{tabular}{|c|c|c|c|c|c|c|c|c|c|c|c|c|}
\hline $\begin{array}{l}\text { I am enjoying spending } \\
\text { more time at home during } \\
\text { the lockdown }\end{array}$ & $\begin{array}{l}5 \text {-point scale, } 1=\text { strongly } \\
\text { disagree to } 5=\text { strongly } \\
\text { agree, } n=2197\end{array}$ & $\mathrm{M}=3.00, \mathrm{SD}=1.03$ & $12 \cdot 15$ & .005 & $.001 * *$ & .07 & $.001 * *$ & $17 \cdot 20$ & .096 & $<.001 * *$ & .06 & $.01 *$ \\
\hline $\begin{array}{l}\text { Because of coronavirus, I } \\
\text { feel a sense of community } \\
\text { with other people in my } \\
\text { neighbourhood }\end{array}$ & $\begin{array}{l}5 \text {-point scale, } 1=\text { strongly } \\
\text { disagree to } 5=\text { strongly } \\
\text { agree, } n=2189\end{array}$ & $\mathrm{M}=3.00, \mathrm{SD}=1.03$ & $36 \cdot 85$ & .016 & $<\cdot 001 * *$ & $\cdot 13$ & $<.001 * *$ & 19.53 & $\cdot 109$ & $<.001 * *$ & $\cdot 13$ & $<\cdot 001 * *$ \\
\hline
\end{tabular}

$$
\text { neighbourhood }
$$

$* p \leq \cdot 05$

$* * p \leq \cdot 001$

$\dagger$ Adjusting for gender, age, having a child in the household, being extremely clinically vulnerable oneself, employment status, highest level of education or professional qualification, indices of multiple deprivation, social grade, living in a rural or urban area, living alone, marital status, and region. Personal and clinical characteristics entered as first block, other independent variables entered as second block. 
medRxiv preprint doi: https://doi.org/10.1101/2020.06.23.20137901; this version posted June 23, 2020. The copyright holder for this preprint (which was not certified by peer review) is the author/funder, who has granted medRxiv a license to display the preprint in perpetuity.

It is made available under a CC-BY 4.0 International license.

\section{DISCUSSION}

Reported rates of probable anxiety and depression in this sample were far higher than prelockdown population norms, with $21.9 \%$ reporting indicators compatible with clinical anxiety and $23.5 \%$ reporting indicators compatible with clinical depression. Normative data indicate that these rates are usually approximately $5 \%$ and $7 \%$, respectively. ${ }^{19}$ The use of wellestablished measures that have normative data from the pre-pandemic period increases the methodological rigour of this study. ${ }^{11}$ As a point of comparison, using the same measure, the English national cohort study of flooding and health found only slightly higher levels of anxiety in their sample who had experienced flooding (28.3\%) and slightly lower levels of depression $(20 \cdot 1 \%) .{ }^{20}$ While we cannot be certain about the absolute rates of anxiety and depression, high rates of psychological ill-health during lockdown have also been reported in other countries ${ }^{5-8}$ as well as in the UK. ${ }^{9,10}$

Anxiety and depression were associated with markers of greater financial hardship, such as not working, living in a more deprived area, and thinking that if you followed the Government advice, it would have negative financial consequences. ${ }^{1,10}$ These findings suggest that tackling financial concerns may decrease psychological distress. Government measures should aim to provide extra support for those at greater risk of negative financial consequences. The negative financial impact of measures put in place to prevent the spread of COVID-19 has been greater among younger people and low-income households. ${ }^{4}$ Measures aiming to help with financial costs of the pandemic have been widely taken up in the UK, with 8.4 million workers having been "furloughed" through the UK Government job retention scheme (over one-quarter of all workers). ${ }^{21}$ However, those affected are likely to be concerned about uncertainties related to their job and future prospects. As the UK moves into the use of contact tracing to help prevent the spread of coronavirus as businesses and schools re-open, it is essential that people continue to be supported. This includes immediate reimbursement for the financial costs of self-isolation if they develop COVID-19 symptoms or are told that they have been in contact with someone who has COVID-19. ${ }^{14}$ While people isolating would be eligible for statutory sick pay if not entitled to a company-wide sick pay scheme ${ }^{22}$ for many this would have a negative financial impact compared to their usual earnings.

Social factors were also associated with psychological distress in our sample. Anxiety and depression were associated with lower perceived social norms, thinking that you would lose 
medRxiv preprint doi: https://doi.org/10.1101/2020.06.23.20137901; this version posted June 23, 2020. The copyright holder for this preprint

touch with family or friends if you followed Government measures, greater conflict with the people you lived with, not enjoying time at home and lower sense of community with your neighbourhood. Other research has also found an association between depression and lower social support, ${ }^{8}$ and it is likely that limited support at least partly underlies our findings. However, the pattern of results also hints at a more corrosive cause, with conflict within households, declining solidarity with neighbours and feeling that others are not obeying the rules all impacting on mental health. As lockdown eases, fractures within communities may be appearing that are affecting morale and generating distress.

Being female and younger were also associated with a greater likelihood of anxiety and depression. Research in the UK and other countries during lockdown measures for COVID19 has also found that females and those who are younger show greater levels of psychological distress. ${ }^{5-9}$ The prevalence of anxiety and depression is generally higher in females than males, ${ }^{23}$ and there is evidence that rates of anxiety and depression decrease with increasing age. ${ }^{24}$ Thus it is unsurprising that these characteristics are associated with anxiety and depression during the lockdown. However, findings from a series of cross-sectional surveys in the UK found that anxiety was higher in women than men in earlier stages of the lockdown, but that this gap narrowed over time. ${ }^{10}$ Evidence suggests that the burden of lockdown measures, especially in families with children, is differentially placed on females. A recent survey of over 3,500 families in the UK found that mothers were spending more time on household responsibilities than fathers, and were more likely to have lost their job, quit their job, or been furloughed. ${ }^{25}$ As schools begin to re-open in England, the burden of increased childcare may begin to ease for some parents, despite controversy around schools re-opening. ${ }^{26}$ Results from this study suggest that targeted communications and increased provision of support for those at greater risk of psychological distress may be warranted.

There was little evidence that illness-related factors, such as thinking that you have had or currently have COVID-19, presence of COVID-19 symptoms in the household, or greater perceived susceptibility of catching COVID-19 were associated with psychological distress. This is reassuring given the large number of people who have caught COVID-19 in the UK, ${ }^{27}$ and who may contract it in the future. However, lower perceived effectiveness of Government measures in preventing the spread of COVID-19 was associated with anxiety and depression. Results suggest that stressors associated with measures put in place to prevent the spread of COVID-19 were associated with psychological distress, rather than stressors associated with the illness itself. Greater worry about COVID-19 was also 
associated with psychological distress (note that there was no longer an association with depression when correcting for multiple comparisons). ${ }^{9}$ While greater worry is associated with increased adherence to lockdown measures, ${ }^{14}$ communications that decrease worry may also promote behaviours such as return to work. ${ }^{28}$ Highlighting that measures are effective is likely to decrease psychological distress, as well as promoting adherence. ${ }^{14}$

Poorer self-reported general health was associated with markers of greater financial hardship, such as living in a more deprived area, lower social grade, and having a chronic illness that makes you clinically extremely vulnerable to severe complications from COVID-19. Similarly, poorer psychological health was associated with poorer self-reported general health. These findings are in line with epidemiological findings indicating that greater inequality is associated with poorer health and wellbeing. ${ }^{29}$ Associations between psychological and situational factors and self-reported general health are difficult to disentangle given the cross-sectional nature of the study. However, results suggest plausible findings: those who reported that they were generally more healthy were more likely to have helped others due to COVID-19, while those who report that they are generally less healthy were more likely to have received help and to report that they were self-isolating.

This study has several limitations. First, while quota sampling was used and data were weighted to increase representativeness of the sample to the UK general population, we cannot be certain that views of survey respondents are representative of views of the general population. ${ }^{30,31}$ However, associations within the data still provide useful insights. ${ }^{12}$ Second, the cross-sectional nature of this study means that we are unable to imply causality. Third, we investigated self-reported general health using the first item of the SF-36, rather than the complete measure. Therefore, we were not able to compare data to population norms. ${ }^{32}$ This decision was taken due to time and space restrictions for the survey. Fourth, we calculated rates of probable anxiety and depression based on scores from self-report scales (GAD-2 and PHQ-2 respectively). However, these scales have been validated and are widely used as screening tools for psychological ill-health. ${ }^{16,17}$ Fifth, we did not measure ethnicity and so cannot say if mental health was differentially affected in different ethnic groups.

While we cannot be certain of the exact prevalence, rates of probable anxiety and depression in the UK were substantially higher during the lockdown than population norms. Most people rated their general health as "good". Data suggest that psychological distress was associated with greater financial hardship, lower social support and greater conflict. Psychological distress was also associated with sociodemographic factors, such as being female, younger, 
medRxiv preprint doi: https://doi.org/10.1101/2020.06.23.20137901; this version posted June 23,2020 . The copyright holder for this preprint (which was not certified by peer review) is the author/funder, who has granted medRxiv a license to display the preprint in perpetuity.

It is made available under a CC-BY 4.0 International license .

unemployed, and living in a more deprived area. Poorer self-reported general health was also associated with greater inequality. These findings highlight the urgent need for better social and financial support for isolated, financially insecure young people to reduce the mental health impact of the pandemic and for greater attention among public health policy makers to the value of familial and community solidarity as potentially protective for mental health. 
medRxiv preprint doi: https://doi.org/10.1101/2020.06.23.20137901; this version posted June 23, 2020. The copyright holder for this preprint (which was not certified by peer review) is the author/funder, who has granted medRxiv a license to display the preprint in perpetuity.

It is made available under a CC-BY 4.0 International license .

\section{FUNDING SOURCES}

LS, RA and GJR are supported by the National Institute for Health Research Health Protection Research Unit (NIHR HPRU) in Emergency Preparedness and Response, a partnership between Public Health England, King's College London and the University of East Anglia. RA, HL, IO, CR and LY are supported by the NIHR HPRU in Behavioural Science and Evaluation, a partnership between Public Health England and the University of Bristol. CR is also supported by the NIHR HPRU in Emerging and Zoonotic Infections and NIHR HPRU in Gastrointestinal Infections. The views expressed are those of the authors and not necessarily those of the UKRI, NIHR, Public Health England or the Department of Health and Social Care. The NIHR HPRU Emergency Preparedness and Response funded the study. An MRC award under the MRC COVID-19 Rapid Response call (grant number MC_PC_19071) funded RA, HL, IO, CR, LY and GJR's time.

\section{DATA SHARING STATEMENT}

Anonymised data will be made available upon reasonable request. 
medRxiv preprint doi: https://doi.org/10.1101/2020.06.23.20137901; this version posted June 23, 2020. The copyright holder for this preprint (which was not certified by peer review) is the author/funder, who has granted medRxiv a license to display the preprint in perpetuity.

It is made available under a CC-BY 4.0 International license .

\section{REFERENCES}

1. Brooks SK, Webster RK, Smith LE, et al. The psychological impact of quarantine and how to reduce it: rapid review of the evidence. Lancet 2020; 395(10227): 912-20.

2. Cabinet Office. New rules on staying at home and away from others, 2020.

3. HM Government. Our plan to rebuild: The UK Government's COVID-19 recovery strategy, 2020.

4. Blundell R, Costa Dias M, Joyce R, Xu X. COVID-19 and inequalities, 2020.

5. Madani A, Boutebal SE, Bryant CR. The Psychological Impact of Confinement Linked to the Coronavirus Epidemic COVID-19 in Algeria. Int J Environ Res Public Health 2020; 17(10).

6. Mazza C, Ricci E, Biondi S, et al. A Nationwide Survey of Psychological Distress among Italian People during the COVID-19 Pandemic: Immediate Psychological Responses and Associated Factors. Int J Environ Res Public Health 2020; 17(9): 3165.

7. Prati G. Mental Health and Its Psychosocial Predictors during National Quarantine in Italy against the Corona Virus Disease 2019 (COVID $\square$ 19). PsyArXiv 2020.

8. Tull MT, Edmonds KA, Scamaldo KM, Richmond JR, Rose JP, Gratz KL.

Psychological Outcomes Associated with Stay-at-Home Orders and the Perceived Impact of COVID-19 on Daily Life. Psychiatry Res 2020; 289: 113098.

9. Jia R, Ayling K, Chalder T, et al. Mental health in the UK during the COVID-19

pandemic: early observations MedRxiv 2020.

10. Office for National Statistics. Coronavirus and anxiety, Great Britain: 3 April 2020 to 10 May 2020, 2020.

11. Pierce M, McManus S, Jessop C, et al. Says who? The significance of sampling in mental health surveys during COVID-19. Lancet Psychiatry 2020.

12. Kohler U. Possible Uses of Nonprobability Sampling for the Social Sciences. Survey Methods: Insights from the Field 2019.

13. Rubin GJ, Amlot R, Page L, Wessely S. Methodological challenges in assessing general population reactions in the immediate aftermath of a terrorist attack. Int J Methods Psychiatr Res 2008; 17 Suppl 2: S29-35.

14. Smith LE, Amlot R, Lambert H, et al. Factors associated with adherence to selfisolation and lockdown measures in the UK; a cross-sectional survey. Public Health Submitted.

15. Rubin GJ, Smith LE, Melendez-Torres GJ, Yardley L. Improving adherence to 'Test, Trace and Isolate'. BMJ Submitted.

16. Kroenke K, Spitzer RL, Williams JB, Monahan PO, Lowe B. Anxiety disorders in primary care: prevalence, impairment, comorbidity, and detection. Ann Intern Med 2007; 146(5): 317-25.

17. Kroenke K, Spitzer RL, Williams JB. The Patient Health Questionnaire-2: validity of a two-item depression screener. Med Care 2003; 41(11): 1284-92.

18. Ware JE, Jr., Sherbourne CD. The MOS 36-item short-form health survey (SF-36). I. Conceptual framework and item selection. Med Care 1992; 30(6): 473-83.

19. Lowe B, Wahl I, Rose M, et al. A 4-item measure of depression and anxiety: validation and standardization of the Patient Health Questionnaire-4 (PHQ-4) in the general population. J Affect Disord 2010; 122(1-2): 86-95.

20. Waite TD, Chaintarli K, Beck CR, et al. The English national cohort study of flooding and health: cross-sectional analysis of mental health outcomes at year one. BMC Public Health 2017; 17(1): 129.

21. Andy Gregory. Coronavirus: Nearly one in four UK workers furloughed in past fortnight, government data shows. The Guardian. 20205 May 2020. 
medRxiv preprint doi: https://doi.org/10.1101/2020.06.23.20137901; this version posted June 23, 2020. The copyright holder for this preprint (which was not certified by peer review) is the author/funder, who has granted medRxiv a license to display the preprint in perpetuity.

It is made available under a CC-BY 4.0 International license .

22. HM Government. Statutory Sick Pay (SSP). 2020. https://www.gov.uk/statutory-sickpay (accessed 22 June 2020).

23. McLean CP, Asnaani A, Litz BT, Hofmann SG. Gender differences in anxiety disorders: prevalence, course of illness, comorbidity and burden of illness. J Psychiatr Res 2011; 45(8): 1027-35.

24. Jorm AF. Does old age reduce the risk of anxiety and depression? A review of epidemiological studies across the adult life span. Psychol Med 2000; 30(1): 11-22.

25. Andrew A, Cattan S, Costa Dias M, et al. How are mothers and fathers balancing work and family under lockdown? The Institute for Fiscal Studies, 2020.

26. Richard Adams. Coronavirus: Schools expect half of pupils will stay home as year groups return. The Guardian. 19 May 2020.

27. Public Health England. Coronavirus (COVID-19) in the UK. 1 June 2020. Available from: https://coronavirus.data.gov.uk/? ga=2.173670241.393565602.1591027602-

786651327.1579191761 (accessed 2 June 2020).

28. Rubin GJ, Potts HWW, Michie S. The impact of communications about swine flu (influenza A H1N1v) on public responses to the outbreak: results from 36 national telephone surveys in the UK. Health Technol Asses 2010; 14(34): 183-266.

29. Pickett KE, Wilkinson RG. Income inequality and health: a causal review. Soc Sci Med 2015; 128: 316-26.

30. Wright KB. Researching Internet-Based Populations Advantages and Disadvantages of Online Survey Research, Online Questionnaire Authoring Software Packages, and Web Survey Services. Journal of Computer-Mediated Communication, 2005.

31. Office for National Statistics. Internet users, UK: 2019, 2019.

32. Bowling A, Bond M, Jenkinson C, Lamping DL. Short Form 36 (SF-36) Health Survey questionnaire: which normative data should be used? Comparisons between the norms provided by the Omnibus Survey in Britain, the Health Survey for England and the Oxford Healthy Life Survey. J Public Health Med 1999; 21(3): 255-70. 